\title{
Nash equilibria in noncooperative predator-prey games
}

\author{
Ángel Manuel Ramos ${ }^{1}$, Tomáš Roubíček ${ }^{2,3}$ \\ ${ }^{1}$ Corresponding author, \\ Departamento de Matemática Aplicada, Universidad Complutense de Madrid, \\ Plaza de Ciencias 3, 28040, Madrid, Spain. \\ Angel_Ramos@mat.ucm.es \\ 2 Mathematical Institute, Charles University, \\ Sokolovská 83, CZ-186 75 Praha 8, Czech Republic, \\ roubicek@karlin.mff.cuni.cz \\ 3 Institute of Information Theory and Automation, Academy of Sciences, \\ Pod vodárenskou věží 4, CZ-182 08 Praha 8, Czech Republic.
}

\begin{abstract}
A non-cooperative game governed by a distributed-parameter predator-prey system is considered, assuming that two players control initial conditions for predator and prey, respectively. Existence of a Nash equlibrium is shown under the condition that the desired population profiles and the environmental carrying capacity for the prey are sufficiently small. A conceptual approximation algorithm is proposed and analyzed. Finally, numerical simulations are performed, too.
\end{abstract}

Keywords: Reaction-diffusion system, Lotka-Volterra system, Nash equilibrium, 2nd-order analysis, existence, numerical simulations.

AMS Classification: 49N70, 91A10, 35Q80.

\section{INTRODUCTION}

Mathematical models in ecological systems represent a long-time scrutinized area. In a distributed-parameter version, they are essentially reaction-diffusion systems with righthand sides of certain special type forming the so-called Lotka-Volterra system. Original studies of oscillation in biological or ecological systems (not necessarily in the presence of diffusion) has been started by Lotka [14] and Volterra [28]. Depending on increasing/decreasing behavior, this includes also the predator-prey system. To be more specific we will use the model involving possibly a satiation, as used already e.g. by Freedman [8], May [15], or Huang, Lu, Ruan [11], cf. also [16, Sect.3.3] for an ODE-variant. Let us emphasize that the model with satiation is very realistic because it reflects the universal phenomenon that predators essentially do not hunt prey unless being hungry or being threatened by hunger; humans are an exception but even their feeding capacity is limited. Anyhow, in mathematical literature one can find both models with satiation and simplified models without satiation, cf. e.g. Britton [2], Murray [16, Sect.12.2], Pao [19, Sect.12.4], or also [27, Sect.12.3] or [29, System (4.1)] etc.. For this reason we shall formulate our results to cover also no-satiation case. More general nonlinearities that those we will employ in (1) can also be found, cf. e.g. [13, 29].

When having modelled such ecological systems, a natural (sometimes unfortunate) ambition of mankind is to control them. This is typically much more difficult than mere 
modelling. An important phenomenon which can be exploited in various ways is convexity of the cost functional. This is, however, not always guaranteed because the controlled predator/prey ecological system is naturally nonlinear.

In this paper, we investigate this convexity property in a nontrivial context of noncooperative games where it is essential to ensure a mere existence of Nash equilibria. It should be, however, remarked that the results concerning convexity analysis can be directly exploited in conventional optimal control where it induces, e.g., sufficiency of 1st-order optimality conditions or global convergence of optimization routines to globally optimal solutions.

In Section 2, we specify the predator/prey system and derive basic properties we will need further. In Section 3 we specify the noncooperative game, perform the abovementioned convexity analysis, and eventually show existence of its Nash equilibria. Section 4 deals with a conceptual discretization of the game, namely a time discretization of the controlled predator/prey system and spatial discretization of the control variables, and prove some sort of convergence. In Section 5 we introduced the full discretization case and eventually, Section 6 presents an illustrative numerical example.

\section{The CONTROlled STATE SYSTEM}

We will consider a fixed bounded Lipschitz domain $\Omega \subset \mathbb{R}^{\mathrm{n}}$ where the game between the predator species and the prey takes place, $n \leq 3$ (although in most ecological applications $n=2$ can be supposed), and $T>0$ a finite time horizon. We denote $Q:=(0, T) \times \Omega$ and $\Sigma:=(0, T) \times \Gamma$, with $\Gamma:=\partial \Omega$ the boundary of $\Omega$. We consider the following predator-prey model:

$$
\begin{array}{ll}
\frac{\partial y}{\partial t}-d_{1} \Delta y=a y\left(1-\frac{y^{+}}{k}\right)-b \frac{y z^{+}}{1+e y^{+}} & \text {in } Q, \\
\frac{\partial z}{\partial t}-d_{2} \Delta z=-c z+d \frac{y z}{1+e y^{+}} & \text {in } Q, \\
y=0, \quad z=0 & \text { on } \Sigma, \\
y(0, \cdot)=u, \quad z(0, \cdot)=v & \text { in } \Omega,
\end{array}
$$

where $(\cdot)^{+}$denotes the non-negative part and, in fact, is only to avoid formal difficulties in some proofs and is out of any effects for non-negative solutions in which we will be interested so we are definitely authorized to define the right-hand side nonlinearities in a whatever way we need. The unknown functions $y=y(t, x)$ and $z=z(t, x)$ are the densities of the prey and the predator species, while the given functions $u=u(x) \geq 0$ and $v=v(x) \geq 0$ are the initial densities of them, respectively. The initial settings $u$ and $v$ of the species into the environment area $\Omega$ will later be used as controls. The constants in (1) have the following meaning:

$d_{1}, d_{2}>0$ are the diffusion coefficient related with migration,

$a>0$ a growth factor for the prey species,

$k>0$ the carrying capacity,

$c>0$ a death rate for the predator in the absence of the prey species,

$b, d>0$ the interaction rates, 
$e \geq 0$ a parameter determining a satiation rate.

We use the standard notation for Lebesgue spaces $L^{p}(\Omega)$ of measurable functions on $\Omega$ whose $p$-power is integrable. Further, as usual, $H_{0}^{1}(\Omega)$ denotes the Sobolev space of functions whose distributional derivatives belong to $L^{2}(\Omega)$ and whose traces on $\Gamma$ vanishes; let us agree that the norm in $H_{0}^{1}(\Omega)$ is $\|y\|_{H_{0}^{1}(\Omega)}:=\|\nabla y\|_{L^{2}\left(\Omega ; \mathbb{R}^{\mathrm{n}}\right)}$. We will also use the Bochner spaces $L^{p}(0, T ; X)$ of functions $[0, T] \rightarrow X$ measurable in Bochner's sense whose $p$-power is integrable and we will denote

$$
\mathcal{W}:=\left\{y \in L^{2}\left(0, T ; H_{0}^{1}(\Omega)\right) ; \quad \frac{\partial y}{\partial t} \in L^{2}\left(0, T ; H^{-1}(\Omega)\right)\right\} .
$$

The conventional norms in the space $X$ in question will be denoted by $\|\cdot\|_{X}$, but instead of $\|\cdot\|_{L^{p}(\Omega)}$ we will write shortly $\|\cdot\|_{p}$.

Proposition 2.1. Assume $0 \leq u \leq k, 0 \leq v \leq K$ a.e. in $\Omega$. Then system (1) possesses a unique solution $(y, z) \in L^{\infty}(Q)^{2} \cap \mathcal{W}^{2}$. To be more specific, it holds

$$
0 \leq y \leq k, \quad 0 \leq z \leq \mathrm{e}^{\left(\frac{d k}{1+e k}-c\right)^{+} T} K \quad \text { a.e. in } Q
$$

and

$$
0 \leq z(t) \leq \mathrm{e}^{\left(\frac{d k}{1+e k}-c\right) t} K \quad \text { a.e. in } \Omega, \forall t \in[0, T] .
$$

Moreover, the dependence $(u, v) \mapsto(y, z): L^{2}(\Omega)^{2} \rightarrow L^{2}(Q)^{2}$ is (weak,norm)-continuous.

Proof. Existence of a weak solution $(y, z)$ to (1) can be proved, e.g., by a constructive way by making a semi-explicit semi-discretization in time and a limit passage, as done in Sect. 4 below. Let us now investigate its qualitative properties we will need later.

Now we prove that each weak solution (not only those obtained as a limit mentioned above) is non-negative. Indeed, by testing (1a) by $y^{-}$, we get

$$
\frac{1}{2} \frac{\mathrm{d}}{\mathrm{d} t}\left\|y^{-}\right\|_{2}^{2}+d_{1}\left\|\nabla y^{-}\right\|_{2}^{2}+b \int_{\Omega} \frac{y y^{-} z^{+}}{1+e y^{+}} \mathrm{d} x=a\left\|y^{-}\right\|_{2}^{2} ;
$$

note that $a y y^{+} y^{-} / k=0$. From (4) we get $\left\|y^{-}\right\|_{2} \leq 0$ by Gronwall's inequality because $\left\|u^{-}\right\|_{2}=0$ and the integral in the left-hand side of (4) is non-negative. Hence $y \geq 0$ a.e. on $Q$.

Now, by testing $(1 \mathrm{a})$ by $(y-k)^{+}$, we get

$$
\frac{1}{2} \frac{\mathrm{d}}{\mathrm{d} t}\left\|(y-k)^{+}\right\|_{2}^{2}+d_{1}\left\|\nabla(y-k)^{+}\right\|_{2}^{2}+\int_{\Omega} a y \frac{y^{+}-k}{k}(y-k)^{+}+b \frac{y(y-k)^{+} z^{+}}{1+e y^{+}} \mathrm{d} x=0,
$$

from which we get $\left\|(y-k)^{+}\right\|_{2} \leq 0$ by Gronwall's inequality because $\left\|(u-k)^{+}\right\|_{2}=0$ and the integral in the left-hand side of $(5)$ is non-negative. Hence $y \leq k$ a.e. in $Q$. Note that the $L^{\infty}$-bound, namely $0 \leq y \leq k$, is independent of $z$ and that it holds for any weak solution, not only that one obtained as a limit in Sect. 4.

Now we will prove $z(t, x) \geq 0$ a.e. on $\Omega$ by testing (1b) by $z^{-}$. This gives:

$$
\frac{1}{2} \frac{\mathrm{d}}{\mathrm{d} t}\left\|z^{-}\right\|_{2}^{2}+d_{2}\left\|\nabla z^{-}\right\|_{2}^{2}+c\left\|z^{-}\right\|_{2}^{2}=d \int_{\Omega} \frac{y z z^{-}}{1+e y^{+}} \mathrm{d} x \leq d \frac{k}{1+e k}\left\|z^{-}\right\|_{2}^{2}
$$

note that we used $0 \leq y \leq k$ which has been already proved. From (6) we get $\left\|z^{-}\right\|_{2} \leq 0$ by Gronwall's inequality because $\left\|v^{-}\right\|_{2}=0$. Hence $z \geq 0$ a.e. in $Q$. 
By a substitution $Z=\mathrm{e}^{-\lambda t} z$, the equation in (1b) transforms to

$$
\frac{\partial Z}{\partial t}-d_{2} \Delta Z=\left(d \frac{y}{1+e y^{+}}-c-\lambda\right) Z
$$

For $\lambda=\left(\frac{d k}{1+e k}-c\right)$, we get $Z(t) \leq\|v\|_{\infty}$ a.e. in $\Omega$, so that

$$
z(t) \leq \mathrm{e}^{\left(\frac{d k}{1+e k}-c\right) t}\|v\|_{\infty} \quad \text { a.e. in } \Omega, \quad \forall t \in[0, T]
$$

and

$$
z \leq \mathrm{e}^{\left(\frac{d k}{1+e k}-c\right)^{+} T}\|v\|_{\infty} \quad \text { a.e. in } Q .
$$

Again note that (8) holds for any weak solution, not only that one obtained as a limit in Sect. 4 .

To prove uniqueness of the solution to (1), we consider two weak solutions $\left(y_{1}, z_{1}\right)$ and $\left(y_{2}, z_{2}\right)$ to the initial-boundary-value problem (1), subtract the corresponding equations and test them by $y_{1}-y_{2}$ and $z_{1}-z_{2}$, respectively. This gives

$$
\begin{aligned}
& \frac{1}{2} \frac{\mathrm{d}}{\mathrm{d} t}\left(\left\|y_{1}-y_{2}\right\|_{2}^{2}\right.\left.+\left\|z_{1}-z_{2}\right\|_{2}^{2}\right)+d_{1}\left\|\nabla\left(y_{1}-y_{2}\right)\right\|_{2}^{2} \\
&+d_{2}\left\|\nabla\left(z_{1}-z_{2}\right)\right\|_{2}^{2}+\int_{\Omega} \frac{a}{k}\left(y_{1}+y_{2}\right)\left(y_{1}-y_{2}\right)^{2} \mathrm{~d} x+c\left\|z_{1}-z_{2}\right\|_{2}^{2} \\
&=a\left\|y_{1}-y_{2}\right\|_{2}^{2}-\int_{\Omega} b\left(\frac{y_{1} z_{1}}{1+e y_{1}}-\frac{y_{2} z_{2}}{1+e y_{2}}\right)\left(y_{1}-y_{2}\right) \\
&+d\left(\frac{y_{1} z_{1}}{1+e y_{1}}-\frac{y_{2} z_{2}}{1+e y_{2}}\right)\left(z_{1}-z_{2}\right) \\
& \leq a\left\|y_{1}-y_{2}\right\|_{2}^{2}+b\left\|y_{1}\right\|_{\infty}\left\|z_{1}-z_{2}\right\|_{2}\left\|y_{1}-y_{2}\right\|_{2}+b\left\|y_{1}-y_{2}\right\|^{2}\left\|z_{2}\right\|_{\infty} \\
&+d\left\|y_{1}\right\|_{\infty}\left\|z_{1}-z_{2}\right\|_{2}^{2}+d\left\|y_{1}-y_{2}\right\|_{2}\left\|z_{2}\right\|_{\infty}\left\|z_{1}-z_{2}\right\|_{2},
\end{aligned}
$$

where we also used that the function $y \mapsto y /(1+e y):[0,+\infty] \rightarrow \mathbb{R}$ is Lipschitz with the derivative bounded by 1 , that $y_{1}, y_{2}, z_{1}$, and $z_{2}$ are non-negative and that $y_{1}$ and $z_{2}$ have upper bounds. Then, by Young's and Gronwall's inequalities, we get $y_{1}-y_{2}=0$ and $z_{1}-z_{2}=0$.

The (weak,weak)-continuous dependence of $(y, z)$ on the initial conditions $(u, v)$ as a mapping $L^{2}(\Omega)^{2} \rightarrow \mathcal{W}^{2}$ follows easily from the obtained a-priori estimates and the uniqueness. Then Aubin-Lions' theorem gives the compact embedding into $L^{2}(Q)$ and thus the claimed mode of continuity.

\section{THE GAME}

We will consider a two-player noncooperative game with the predator-prey system (1). Each player is to control an initial condition in one of the equations (1a,b), having his/her own goals expressed by the desired response as well as the cost of the species controlled by this player. Let us agree that the 1st player will control the initial condition for the prey while the 2 nd player will control the predator and $u_{\max } \geq 0, v_{\max } \geq 0$ will denote the maximal allowed density of the initial setting of the prey and the predator, respectively; the dependence of $u_{\max }$ and $u_{\min }$ on $x$ may reflect territorial authorization for particular players. Our game will have the form: 


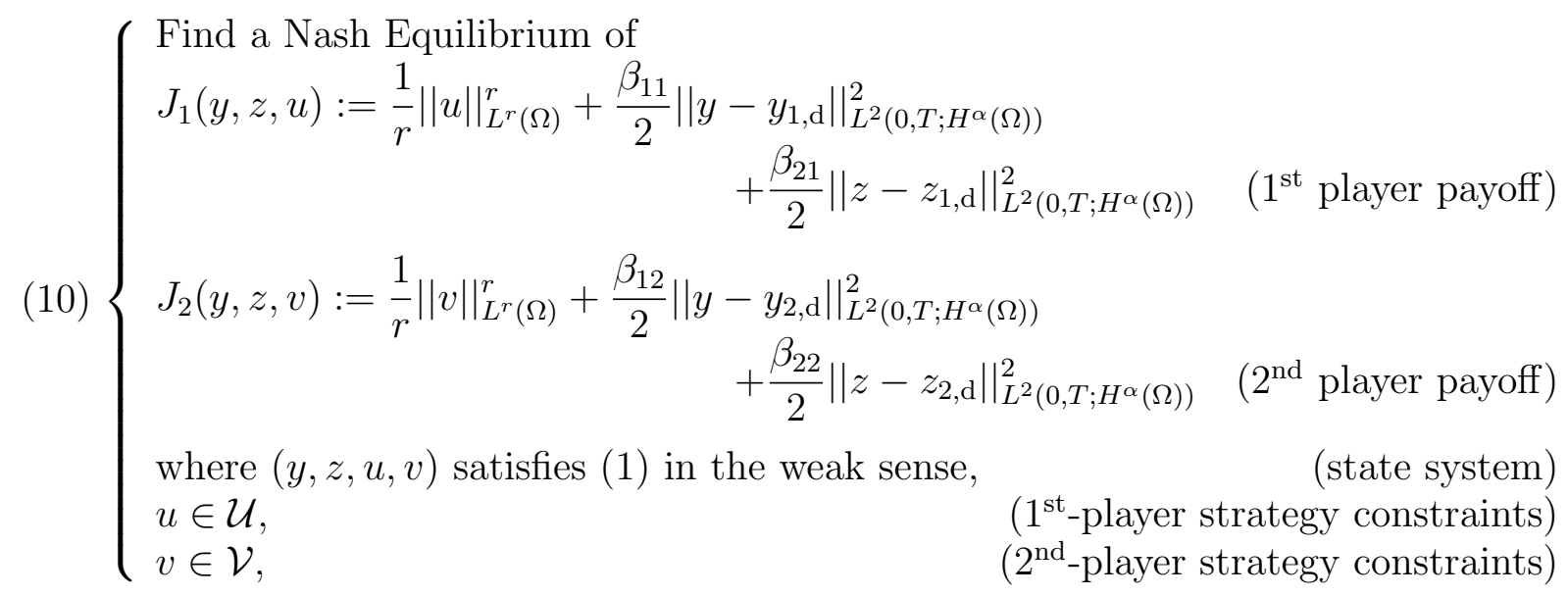

where

$$
\mathcal{U}:=\left\{u \in L^{\infty}(\Omega) ; \quad 0 \leq u \leq u_{\max }\right\}, \quad \mathcal{V}:=\left\{v \in L^{\infty}(\Omega) ; \quad 0 \leq v \leq v_{\max }\right\} .
$$

Remark 3.1. The functionals $J_{1}$ and $J_{2}$ chosen correspond to stabilization problems (see, for instance, [10] for other numerical examples of stabilization type problems). Other type of problems that could have been considered are controllability type problems (see, for instance, [5], [6] and [7])) when we want to minimize the distance of our state solution at the final time to a target state.

We remind (see Proposition 2.1) that $y, z \in \mathcal{W}^{2} \cap L^{\infty}(Q)^{2}$. We are supposing either $r=1$ or $r=2$, so that the respective terms in $J_{1}$ and $J_{2}$ represent a cost of the the species to be initially "bought" or the usual $L^{2}$-norm as conventionally used in mathematical setting, respectively. The notation $H^{\alpha}(\Omega)$ refers to the usual Sobolev-Slobodetskiu space normed by

$$
\|y\|_{H^{\alpha}(\Omega)}:=\left(\int_{\Omega}\left(y(\xi)^{2}+\alpha \int_{\Omega} \frac{|y(x)-y(\xi)|^{2}}{|x-\xi|^{n+2 \alpha}} \mathrm{d} x\right) \mathrm{d} \xi\right)^{1 / 2} ;
$$

in comparison with the conventional setting, we multiply the double-integral term in (12) by $\alpha$, which yields automatically $\|\cdot\|_{H^{\alpha}(\Omega)}=\|\cdot\|_{2}$ if $\alpha=0$. The functions

$$
y_{\ell, \mathrm{d}}, z_{\ell, \mathrm{d}} \in L^{2}\left(0, T ; H^{2 \alpha+\epsilon}(\Omega)\right), \quad \epsilon>0,
$$

are desired profiles of the prey and the predator species from the viewpoint of the $\ell$-th player. For $\alpha=0$, the payoffs $J_{1}$ and $J_{2}$ involve the conventional $L^{2}(Q)$-distance from these desired profiles, while for $\alpha>0$ we take into account also some spatially nonlocal interactions; let us point out that one can still admit sharp spatial interfaces in $y_{\ell, \mathrm{d}}$ and $z_{\ell, \mathrm{d}}$ satisfying (13) if $\alpha<1 / 4$. We will employ this $\alpha$ advantageously to compensate nonlinearity of the controlled system.

As standard, a Nash equilibrium $\left(u^{*}, v^{*}\right)$ is understood to satisfy

$$
\Phi_{1}\left(u^{*}, v^{*}\right)=\min _{u \in \mathcal{U}} \Phi_{1}\left(u, v^{*}\right) \quad \& \quad \Phi_{2}\left(u^{*}, v^{*}\right)=\min _{v \in \mathcal{V}} \Phi_{2}\left(u^{*}, v\right)
$$

where we denote

$$
\Phi_{1}(u, v):=J_{1}(y, z, u), \quad \Phi_{2}(u, v):=J_{2}(y, z, v)
$$

with $y=y(u, v)$ and $z=z(u, v)$ solving (1). 
Usual arguments leading to existence of Nash equilibria always need convexity of the payoff functionals $\Phi_{1}(\cdot, v)$ and $\Phi_{2}(u, \cdot)$. This convexity is, however, not automatic because the controlled system is nonlinear, but it may really hold if this nonlinearity is not dominant (i.e. if $a, b$ and $d$ are small enough) and is compensated by uniform continuity of the pay-offs.

We will prove this convexity by a second-order analysis of the payoffs. This technique was essentially exposed in [9] to establish sufficiency of first-order optimality conditions; see also [26] or, in the context of noncooperative games, also [24, 25]. Let us introduce the Lagrange function for the $\ell$-th player:

$$
L_{\ell}:\left(\mathcal{W} \cap L^{\infty}(Q)\right)^{2} \times \mathcal{U} \times \mathcal{V} \times\left(L^{\infty}\left(0, T ; H_{0}^{1}(\Omega)\right) \cap H^{1}\left(0, T ; L^{2}(\Omega)\right)\right)^{2} \longrightarrow \mathbb{R},
$$

defined, for $y \geq 0$, by

$$
\begin{gathered}
L_{\ell}(y, z, u, v, p, q):=\frac{\beta_{1 \ell}}{2}\left\|y-y_{\ell, \mathrm{d}}\right\|_{L^{2}\left(0, T ; H^{\alpha}(\Omega)\right)}^{2}+\frac{\beta_{2 \ell}}{2}\left\|z-z_{\ell, \mathrm{d}}\right\|_{L^{2}\left(0, T ; H^{\alpha}(\Omega)\right)}^{2} \\
+\int_{\Omega}\left((2-\ell) \frac{1}{r} u^{r}+(\ell-1) \frac{1}{r} v^{r}+u p(0)+v q(0)-y(T) p(T)-z(T) q(T)\right) d x \\
+\int_{Q}\left(\frac{\partial p}{\partial t} y+\frac{\partial q}{\partial t} z-d_{1} \nabla p \cdot \nabla y+p\left(a y\left(1-\frac{y}{k}\right)-b \frac{y z}{1+e y}\right)\right. \\
\left.-d_{2} \nabla q \cdot \nabla z+q\left(-c z+d \frac{y z}{1+e y}\right)\right) \mathrm{d} x \mathrm{~d} t .
\end{gathered}
$$

The adjoint system for the $\ell$-th player is $\left[L_{\ell}\right]_{(y, z)}^{\prime}(y, z, u, v, p, q)=0$. Let us denote its solutions by $p=p_{\ell}$ and $q=q_{\ell}$. Hence, in the classical formulation, $p_{\ell}$ and $q_{\ell}$ are to solve the following system of linear parabolic equations with boundary and terminal conditions:

$$
\begin{aligned}
& -\frac{\partial p_{\ell}}{\partial t}-d_{1} \Delta p_{\ell}=a\left(1-\frac{2}{k} y\right) p_{\ell}+z \frac{\left(d q_{\ell}-b p_{\ell}\right)}{(1+e y)^{2}}+\beta_{1 \ell} D^{2 \alpha}\left(y-y_{\ell, \mathrm{d}}\right) \quad \text { on } Q \text {, } \\
& -\frac{\partial q_{\ell}}{\partial t}-d_{2} \Delta q_{\ell}=-c q_{\ell}-y \frac{b p_{\ell}-d q_{\ell}}{1+e y}+\beta_{2 \ell} D^{2 \alpha}\left(z-z_{\ell, \mathrm{d}}\right) \quad \text { on } Q \\
& p_{\ell}=0, \quad q_{\ell}=0 \quad \text { on } \Sigma, \\
& p_{\ell}(T, \cdot)=0, \quad q_{\ell}(T, \cdot)=0 \quad \text { on } \Omega,
\end{aligned}
$$

where the linear operator $D^{2 \alpha}: H^{\alpha}(\Omega) \rightarrow H^{\alpha}(\Omega)^{*}$ denotes the Gâteaux differential of the quadratic form $y \mapsto\|y\|_{H^{\alpha}(\Omega)}^{2}: H^{\alpha}(\Omega) \rightarrow \mathbb{R}$. We have an explicit formula for the operator of the "fractional derivative", namely

$$
\left[D^{\mu} y\right](x)=2 y(x)+2 \mu \int_{\Omega} \frac{y(x)-y(\xi)}{|x-\xi|^{n+\mu}} \mathrm{d} \xi
$$

cf. [1, Formula (2.14)]. Then, by the standard adjoint-equation technique, we have the formula for the Gâteaux differential of the payoffs:

$$
\left[\Phi_{\ell}\right]^{\prime}(u, v)=\left[L_{\ell}\right]_{(u, v)}^{\prime}\left(y, z, u, v, p_{\ell}, q_{\ell}\right)=\left((2-\ell) u^{r-1}+p_{\ell}(0),(\ell-1) v^{r-1}+q_{\ell}(0)\right)
$$

with $(y, z)$ being the response on $(u, v)$ in question and $\left(p_{\ell}, q_{\ell}\right)$ solves $(17)$. 
Remark 3.2. (Omitting non-smoothness.) The important fact is that the controlled system (1), hence implicitly also each payoff $\Phi_{\ell}$, involves nonlinearities which are nonsmooth in the arguments $y$ and $z$ at zero. Hence the formula (18) holds if $y, z>0$ while in the opposite case it should be understood only to yield a formula for a relevant directional derivative, but this is just what plays role. This is also why we allow to simplify the Lagrangean (16) and the adjoint system (17) by treating (1) as if $(\cdot)^{+}$were not involved.

Lemma 3.3. (The ADJOINT STATES.) Let $\alpha<1 / 2$ and (13) hold. Then there is a unique $\left(p_{\ell}, q_{\ell}\right) \in H^{1}\left(0, T ; L^{2}(\Omega)\right)^{2} \cap L^{\infty}\left(0, T ; H_{0}^{1}(\Omega)\right)^{2}$ solving (17).

Sketch of the proof. The existence of $p_{\ell h}$ and $q_{\ell h}$ can be proved by standards arguments, based on the a-priori estimate obtained by testing (17a) and (17b) (in some approximate variant, e.g., the Galerkin modification) by $\frac{\partial}{\partial t} p_{\ell}$ and $\frac{\partial}{\partial t} q_{\ell}$, respectively, and then making a limit passage. The mentioned test gives

$$
\begin{aligned}
\left\|\frac{\partial p_{\ell}}{\partial t}\right\|_{2}^{2} & +\left\|\frac{\partial q_{\ell}}{\partial t}\right\|_{2}^{2}-\frac{\mathrm{d}}{\mathrm{d} t}\left(\frac{d_{1}}{2}\left\|\nabla p_{\ell}\right\|_{2}^{2}+\frac{d_{2}}{2}\left\|\nabla q_{\ell}\right\|_{2}^{2}+\frac{c}{2}\left\|q_{\ell}\right\|_{2}^{2}\right) \\
& \leq C\left(\left\|p_{\ell}\right\|_{2}+\left\|q_{\ell}\right\|_{2}\right)\left(\left\|\frac{\partial p_{\ell}}{\partial t}\right\|_{2}+\left\|\frac{\partial q_{\ell}}{\partial t}\right\|_{2}\right) \\
& +\beta_{1 \ell}\left\|D^{2 \alpha}\left(y-y_{\ell, \mathrm{d}}\right)\right\|_{2}\left\|\frac{\partial p_{\ell}}{\partial t}\right\|_{2}+\beta_{2 \ell}\left\|D^{2 \alpha}\left(z-z_{\ell, \mathrm{d}}\right)\right\|_{2}\left\|\frac{\partial q_{\ell}}{\partial t}\right\|_{2}
\end{aligned}
$$

with $C$ a constant depending on $\|y\|_{\infty}$ and $\|z\|_{\infty}$, from which we get by Young's and Gronwall's inequalities the bounds of $p_{\ell}$ and $q_{\ell}$ in $L^{\infty}\left(0, T ; H_{0}^{1}(\Omega)\right) \cap H^{1}\left(0, T ; L^{2}(\Omega)\right)$. Realize that, in (19), $\left\|D^{2 \alpha}\left(y-y_{\ell, \mathrm{d}}\right)\right\|_{2}$ is indeed in $L^{2}(0, T)$ because $y \in L^{2}\left(0, T ; H_{0}^{1}(\Omega)\right)$ and $y_{\ell, \mathrm{d}} \in L^{2}\left(0, T ; H^{2 \alpha+\epsilon}(\Omega)\right)$ with some $\epsilon$ positive, hence also $y-y_{\ell, \mathrm{d}} \in L^{2}\left(0, T ; H^{2 \alpha+\epsilon}(\Omega)\right)$, and then $D^{2 \alpha}\left(y-y_{\ell, \mathrm{d}}\right) \in L^{2}(Q)$ provided $\alpha \leq 1 / 2$ is assumed. The fact that $D^{2 \alpha}$ maps $H^{2 \alpha+\epsilon}(\Omega)$ into $L^{2}(\Omega)$ can be seen simply from the estimate

$$
\begin{gathered}
\int_{\Omega}\left|\int_{\Omega} \frac{v(x)-v(\xi)}{|x-\xi|^{n+2 \alpha}} \mathrm{d} \xi\right|^{2} \mathrm{~d} x=\int_{\Omega}\left|\int_{\Omega} \frac{v(x)-v(\xi)}{|x-\xi|^{n / 2+2 \alpha+\epsilon}} \cdot \frac{1}{|x-\xi|^{n / 2-\epsilon}} \mathrm{d} \xi\right|^{2} \mathrm{~d} x \\
\leq \int_{\Omega}\left(\int_{\Omega} \frac{|v(x)-v(\xi)|^{2}}{|x-\xi|^{n+4 \alpha+2 \epsilon}} \mathrm{d} \xi \int_{\Omega} \frac{1}{|x-\xi|^{n-2 \epsilon}} \mathrm{d} \xi\right) \mathrm{d} x \\
\leq\left(\sup _{x \in \Omega} \int_{\Omega} \frac{1}{|x-\xi|^{n-2 \epsilon}} \mathrm{d} \xi\right) \int_{\Omega \times \Omega} \frac{|v(x)-v(\xi)|^{2}}{|x-\xi|^{n+4 \alpha+2 \epsilon}} \mathrm{d} \xi \mathrm{d} x<+\infty
\end{gathered}
$$

where we used Hölder's inequality and the fact that $\int_{\Omega}|x-\xi|^{2 \epsilon-n} \mathrm{~d} \xi$ is bounded independently of $x \in \Omega$.

The uniqueness can be easily proved in a way similar to that of the uniqueness of solution of $(y, z)$ proved in Proposition 2.1 (the proof is even simpler because of the linearity of system (17)). 
The convexity analysis of $\Phi_{\ell}$ is based on the 2nd-order differential of the Lagrange function, for which we have obviously the following formulae:

$$
\begin{aligned}
& {\left[L_{\ell}\right]_{(y, z)}^{\prime \prime}(y, z, u, v, p, q)(\widetilde{y}, \widetilde{z})^{2} }=\beta_{1 \ell}\|\widetilde{y}\|_{L^{2}\left(0, T ; H^{\alpha}(\Omega)\right)}^{2}+\beta_{2 \ell}\|\widetilde{z}\|_{L^{2}\left(0, T ; H^{\alpha}(\Omega)\right)}^{2} \\
& \int_{Q}-\frac{2 a p}{k} \widetilde{y}^{2}+(b p-d q)\left(\frac{-2 e z}{(1+e y)^{3}} \widetilde{y}^{2}+\frac{2}{(1+e y)^{2}} \widetilde{y} \widetilde{z}\right) \mathrm{d} x \mathrm{~d} t, \\
& {\left[L_{\ell}\right]_{(u, v)}^{\prime \prime}(y, z, u, v, p, q)(\widetilde{u}, \widetilde{v})^{2}=(r-1) \int_{\Omega}(2-\ell)|u|^{r-2} \widetilde{u}^{2}+(\ell-1)|v|^{r-2} \widetilde{v}^{2} \mathrm{~d} x, }
\end{aligned}
$$

provided $y \geq 0$ a.e. on $Q$ and, if $y(t, x)=0$ then $\widetilde{y}(t, x) \geq 0$, cf. Remark 3.2. Note that $\left[L_{\ell}\right]_{(u, v)}^{\prime \prime}(\cdot, \cdot, \cdot, \cdot, p, q)$ is constant for $r=1$ or $r=2$.

Lemma 3.4. (InCRement estimate.) For any $(u, v),(\widetilde{u}, \widetilde{v}) \in \mathcal{U} \times \mathcal{V}$, it holds

$$
\begin{aligned}
\Phi_{\ell}(\widetilde{u}, \widetilde{v})- & \Phi_{\ell}(u, v)-\left[L_{\ell}\right]_{(u, v)}^{\prime}\left(y, z, u, v, p_{\ell}, q_{\ell}\right)(\widetilde{u}-u, \widetilde{v}-v) \\
\geq & \inf _{\substack{0 \leq \widehat{y} \\
0 \leq \widehat{z} \leq \mathrm{e}^{(d k-e)+T}}} \frac{1}{2}\left[L_{\ell}\right]_{(y, z, u, v)}^{\prime \prime}\left(\widehat{y}, \widehat{z}, \cdot, \cdot, \widehat{p}_{\ell}, \widehat{q}_{\ell}\right)(\widetilde{y}-y, \widetilde{z}-z, \widetilde{u}-u, \widetilde{v}-v)^{2}
\end{aligned}
$$

where $\left(y, z, p_{\ell}, q_{\ell}\right)$ is the response to $(u, v)$ and $(\widetilde{y}, \widetilde{z})$ is the response to $(\widetilde{u}, \widetilde{v})$, and $\left(\widehat{y}, \widehat{z}, \widehat{p}_{\ell}, \widehat{q}_{\ell}\right)$ corresponds to $(\widehat{u}, \widehat{v})$.

Proof. By using the state equation (1), the adjoint equation (17), and by-part integration in time, we can see (cf. [24, Lemma 2.3]) that the left-hand side of (21) equals to

$$
\int_{Q} D_{f_{\ell}}(\widetilde{y}, \widetilde{z}, y, z) \mathrm{d} x \mathrm{~d} t+\int_{\Omega} D_{g_{\ell}}(\widetilde{u}, \widetilde{v}, u, v) \mathrm{d} x+\int_{Q} p_{\ell} D_{F}(\widetilde{y}, \widetilde{z}, y, z)+q_{\ell} D_{G}(\widetilde{y}, \widetilde{z}, y, z) \mathrm{d} x \mathrm{~d} t
$$

where $D$ denotes the remainder in the Taylor-expansion formula up to the 1st order term, i.e.

$$
\begin{aligned}
& D_{F}(\widetilde{y}, \widetilde{z}, y, z):=F(\widetilde{y}, \widetilde{z})-F(y, z)-F^{\prime}(y, z)(\widetilde{y}-y, \widetilde{z}-z) \\
& D_{G}(\widetilde{y}, \widetilde{z}, y, z):=G(\widetilde{y}, \widetilde{z})-G(y, z)-G^{\prime}(y, z)(\widetilde{y}-y, \widetilde{z}-z),
\end{aligned}
$$

and $F$ and $G$ are the right-hand sides of (1a) and (1b), respectively, considered with $(\cdot)^{+}$ omitted, i.e. $F(y, z):=a y(1-y / k)-b y z /(1+e y)$ and $G(y, z):=-c z+d y z /(1+e y)$, cf. Remark 3.2. Also, analogous meaning has $D_{f_{\ell}}$ for $f_{\ell}(y, z):=\frac{1}{2} \beta_{1 \ell}\left\|y-y_{\ell, \mathrm{d}}\right\|_{L^{2}\left(0, T ; H^{\alpha}(\Omega)\right)}^{2}+$ $\frac{1}{2} \beta_{2 \ell}\left\|z-z_{\ell, \mathrm{d}}\right\|_{L^{2}\left(0, T ; H^{\alpha}(\Omega)\right)}^{2}$. Hence by a simple algebra

$$
D_{f_{\ell}}(\widetilde{y}, \widetilde{z}, y, z)=\beta_{1 \ell}\|\widetilde{y}-y\|_{L^{2}\left(0, T ; H^{\alpha}(\Omega)\right)}^{2}+\beta_{2 \ell}\|\widetilde{z}-z\|_{L^{2}\left(0, T ; H^{\alpha}(\Omega)\right)}^{2} .
$$

As for $D_{g_{\ell}}$, it involves $g_{\ell}(u, v):=\frac{1}{r}(2-\ell)|u|^{r}+\frac{1}{r}(\ell-1)|v|^{r}$ so that

$$
D_{g_{\ell}}= \begin{cases}0 & \text { for } r=1 \\ (2-\ell)(\widetilde{u}-u)^{2}+(\ell-1)(\widetilde{v}-v)^{2} & \text { for } r=2 .\end{cases}
$$

By Taylor expansion, $\left|D_{G}(\widetilde{y}, \widetilde{z}, y, z)\right| \leq \frac{1}{2} \sup \left|G^{\prime \prime}(\widehat{y}, \widehat{z})\right|\left(|\widetilde{y}-y|^{2}+|\widetilde{z}-z|^{2}\right)$ and similar estimates hold for $\left|D_{F}(\widetilde{y}, \widetilde{z}, y, z)\right|$, where the supreme can be taken over all $(\widehat{y}, \widehat{z})$ that can occur for controls ranging over $\mathcal{U} \times \mathcal{V}$ which, in view of (3) with (11), motivated the choice of "inf" in (21). 
Remark 3.5. (InCREMEnt FORMula.) For the satiation rate $e=0$ and for the considered exponents $r=1$ or $r=2$, the optimization system is bi-quadratic (i.e. has quadratic payoff as well as quadratic nonlinearities in the controlled system) and then $L_{\ell}^{\prime \prime}\left(\cdot, \cdot, \cdot, \cdot, p_{\ell}, q_{\ell}\right)$ is constant and we have a simple identity:

$$
\begin{aligned}
\Phi_{\ell}(\widetilde{u}, \widetilde{v})-\Phi_{\ell}(u, v) & =\left[L_{\ell}\right]_{(u, v)}^{\prime}\left(y, z, u, v, p_{\ell}, q_{\ell}\right)(\widetilde{u}-u, \widetilde{v}-v) \\
& +\frac{1}{2}\left[L_{\ell}\right]_{(y, z, u, v)}^{\prime \prime}\left(\cdot, \cdot, \cdot, \cdot, p_{\ell}, q_{\ell}\right)(\widetilde{y}-y, \widetilde{z}-z, \widetilde{u}-u, \widetilde{v}-v)^{2}
\end{aligned}
$$

see [26, Lemma 8].

Proposition 3.6. (CONVEXITY OF $\Phi_{\ell}$.) Let the constants in (1) be given with the carrying capacity $k$ sufficiently small, and let $\alpha \leq 1 / 2$ satisfies also

$$
\alpha \begin{cases}\geq 0 & \text { if } n=1, \\ >0 & \text { if } n=2, \\ \geq 1 / 4 & \text { if } n=3 .\end{cases}
$$

Then, for any $y_{\ell \mathrm{d}}, z_{\ell \mathrm{d}}, \ell=1,2$, satisfying (13) and being sufficiently small in the norms of $L^{2}\left(0, T ; H^{2 \alpha}(\Omega)\right)$, the functional $\Phi_{\ell}$ is convex on $\mathcal{U} \times \mathcal{V}$ if also $\left\|v_{\max }\right\|_{L^{\infty}(\Omega)}$ is sufficiently small and if naturally $u_{\max }(\cdot) \leq k$ on $\Omega$.

Proof. The assumed smallness of $k, u_{\max }$ and $v_{\max }$ guarantees $y$ and $z$ small in the norm $L^{\infty}(Q)$ (cf. Proposition 2.1 with $K=\left\|v_{\max }\right\|_{L^{\infty}(\Omega)}$ ). We can easily see that $y$ and $z$ can be made small in the norm $L^{2}\left(0, T ; H_{0}^{1}(\Omega)\right)$. Indeed, testing (1a) by $y$, we get

$$
\frac{1}{2} \frac{\mathrm{d}}{\mathrm{d} t}\|y\|_{2}^{2}+d_{1}\|\nabla y\|_{2}^{2} \leq a\|y\|_{2}^{2}
$$

so that by the Gronwall inequality we get $\|y\|_{L^{\infty}\left(0, T ; L^{2}(\Omega)\right)} \leq \sqrt{\mathrm{e}^{2 a T}}|| u \|_{2}$ and then again from (26) we get

$$
\|y\|_{L^{2}\left(0, T ; H_{0}^{1}(\Omega)\right)} \leq \sqrt{\frac{e^{2 a T}}{2 d_{1}}}\|u\|_{2}
$$

which shows that $\|y\|_{L^{2}\left(0, T ; H_{0}^{1}(\Omega)\right)}$ must be small if $\|u\|_{2}$ (which is bounded by $\sqrt{|\Omega|}\|u\|_{\infty}$ ) is small. Similarly, testing (1b) by $z$, one gets

$$
\frac{1}{2} \frac{\mathrm{d}}{\mathrm{d} t}\|z\|_{2}^{2}+d_{2}\|\nabla z\|_{2}^{2}+c\|z\|_{2}^{2} \leq d k\|z\|_{2}^{2}
$$

so that by the Gronwall inequality one gets $\|z\|_{L^{\infty}\left(0, T ; L^{2}(\Omega)\right)} \leq \sqrt{\mathrm{e}^{2 \gamma T}}\|v\|_{2}$ with $\gamma:=$ $(d k-c)^{+}$and then again from $(27)$ we get

$$
\|z\|_{L^{2}\left(0, T ; H_{0}^{1}(\Omega)\right)} \leq \sqrt{\frac{e^{2 \gamma T}}{2 d_{2}}}\|v\|_{2}
$$

which shows that $\|z\|_{L^{2}\left(0, T ; H_{0}^{1}(\Omega)\right)}$ must be small if $\|v\|_{2}$ (which is bounded by $\sqrt{|\Omega|}\|v\|_{\infty}$ ) is small. By the proof of Lemma 3.3, we have $\left(p_{\ell}, q_{\ell}\right)$ bounded also in $L^{\infty}\left(0, T ; H_{0}^{1}(\Omega)\right)$ and then, if also $y_{\ell \mathrm{d}}$ and $z_{\ell \mathrm{d}}$ are sufficiently small, we obtain $p_{\ell}$ and $q_{\ell}$ small in $L^{\infty}\left(0, T ; H_{0}^{1}(\Omega)\right)$, 
cf. (19). In particular, we can assume the mentioned data qualification so strict that

$$
\begin{aligned}
\left\|p_{\ell}\right\|_{L^{\infty}\left(0, T ; H_{0}^{1}(\Omega)\right)} & \leq \frac{1}{2 C\left(2 / k+b+2 b e\|z\|_{\infty}\right)}, \\
\left\|q_{\ell}\right\|_{L^{\infty}\left(0, T ; H_{0}^{1}(\Omega)\right)} & \leq \frac{1}{2 C\left(d+2 d e\|z\|_{\infty}\right)}
\end{aligned}
$$

is satisfied with $C$ a constant from the estimate

$$
\int_{\Omega} p y^{2} \mathrm{~d} x \leq C\|p\|_{H_{0}^{1}(\Omega)}\|y\|_{H^{\alpha}(\Omega)}^{2} .
$$

The estimate (29) holds due to the Hölder inequality with Sobolev embeddings provided $\alpha$ is sufficiently large, which here means (25). Note that, in case $n=3, H_{0}^{1}(\Omega) \subset L^{6}(\Omega)$ and $H^{\alpha}(\Omega) \subset L^{6 /(3-2 \alpha)}(\Omega)$ which, for $\alpha=1 / 4$, is just $L^{12 / 5}(\Omega)$ so that $6^{-1}+(12 / 5)^{-1}+$ $(12 / 5)^{-1}=1$, which explains the last bound in $(25)$.

Then, in view of (20), by using the Young inequality and (28), we can estimate:

$$
\begin{aligned}
& {\left[L_{\ell}\right]_{(y, z)}^{\prime \prime}\left(y, z, u, v, p_{\ell}, q_{\ell}\right)(\widetilde{y}, \widetilde{z})^{2} \geq\left(1-\frac{2 a C}{k}\left\|p_{\ell}\right\|_{L^{\infty}\left(0, T ; H_{0}^{1}(\Omega)\right)}\right.} \\
& \left.\quad-C\left(b\left\|p_{\ell}\right\|_{L^{\infty}\left(0, T ; H_{0}^{1}(\Omega)\right)}+d\left\|q_{\ell}\right\|_{L^{\infty}\left(0, T ; H_{0}^{1}(\Omega)\right)}\right)\left(2 e\|z\|_{\infty}+1\right)\right)\|\widetilde{y}\|_{L^{2}\left(0, T ; H^{\alpha}(\Omega)\right)}^{2} \\
& \quad+\left(1-b C\left\|p_{\ell}\right\|_{L^{\infty}\left(0, T ; H_{0}^{1}(\Omega)\right)}-d C\left\|q_{\ell}\right\|_{L^{\infty}\left(0, T ; H_{0}^{1}(\Omega)\right)}\right)\|\widetilde{z}\|_{L^{2}\left(0, T ; H^{\alpha}(\Omega)\right)}^{2} \geq 0 .
\end{aligned}
$$

Altogether, as always $\left[L_{\ell}\right]_{(u, v)}^{\prime \prime}\left(y, z, u, v, p_{\ell}, q_{\ell}\right)(\widetilde{u}, \widetilde{v})^{2} \geq 0$ because $L_{\ell}\left(y, z, \cdot, \cdot, p_{\ell}, q_{\ell}\right)$ is convex, we can join (18) and (24) to obtain

$$
\Phi_{\ell}(\widetilde{u}, \widetilde{v})-\Phi_{\ell}(u, v)-\left[\Phi_{\ell}^{\prime}(u, v)\right](\widetilde{u}-u, \widetilde{v}-v) \geq 0 .
$$

Replacing the role of $(\widetilde{u}, \widetilde{v})$ and $(u, v)$, we get also

$$
\Phi_{\ell}(u, v)-\Phi_{\ell}(\widetilde{u}, \widetilde{v})-\left[\Phi_{\ell}^{\prime}(\widetilde{u}, \widetilde{v})\right](u-\widetilde{u}, v-\widetilde{v}) \geq 0 .
$$

Summing (31) and (32), we get $\left(\left[\Phi_{\ell}^{\prime}(\widetilde{u}, \widetilde{v})\right]-\left[\Phi_{\ell}^{\prime}(u, v)\right]\right)(\widetilde{u}-u, \widetilde{v}-v) \geq 0$. As $\widetilde{u}, u \in \mathcal{U}$ and $\widetilde{v}, v \in \mathcal{V}$ are arbitrary, we have thus proved monotonicity of $\Phi_{\ell}^{\prime}$ on $\mathcal{U} \times \mathcal{V}$. From this, convexity of $\Phi_{\ell}$ follows by a standard argument.

Existence of Nash equilibria [17] by the standard technique developed by Nikaidô and Isoda [18] would require the payoffs (separately) continuous with respect to compact topologies on $\mathcal{U}$ and $\mathcal{V}$, which is not compatible with usual norm or weak topologies on these sets if $r \neq 1$. Alternatively, we could make a relaxation by means of Young measures, prove existence in terms of mixed strategies, and then to show from the maximum principle that each Nash equilibrium must be, in fact, composed from pure strategies (ranging over the original sets $\mathcal{U}$ and $\mathcal{V}$ ), cf. [23, Chapter 7] for this approach. We, however, choose even a simpler technique based on the Schauder-type fixed-point theorem, which is even slightly more constructive than [18] where, beside the Schauder theorem, also a non-constructive selection by compactness argument and a contradiction argument are used.

Proposition 3.7. (EXISTence of NASH Equilibria.) Under the conditions of Proposition 3.6, the game (10) possesses at least one Nash equilibrium. 
Proof. Any fixed point on $\mathcal{U} \times \mathcal{V}$ of the set-valued mapping

$$
(u, v) \mapsto\left(\operatorname{Argmin}_{\mathcal{U}} \Phi_{1}(\cdot, v), \operatorname{Argmin}_{\mathcal{V}} \Phi_{2}(u, \cdot)\right)
$$

where $\operatorname{Argmin}_{\mathcal{U}} \Phi_{1}(\cdot, v):=\left\{u \in \mathcal{U} ; \Phi_{1}(u, v)=\min \Phi_{1}(\mathcal{U}, v)\right\}$ and $\operatorname{Argmin}_{\mathcal{V}} \Phi_{2}(u, \cdot)$ being defined analogously, is obviously a Nash equilibrium.

We will prove existence of such fixed point by Schauder-type theorem. As the inequalities in (28) are not strict, we do not have strict convexity of the payoffs, hence (33) can indeed be a set-valued mapping in general, and we are to use Kakutani's generalization of Schauder's theorem, which needs $\mathcal{U} \times \mathcal{V}$ to be convex and compact, and the mapping (33) to be upper semicontinuous with closed convex values. These requirements are valid if a weak ${ }^{*} L^{\infty}(\Omega)$ topology is taken on both $\mathcal{U}$ and $\mathcal{V}$. Indeed, the convexity of $\operatorname{Argmin}_{\mathcal{U}} \Phi_{1}(\cdot, v)$ follows from the convexity of $\mathcal{U}$ and of $\Phi_{1}(\cdot, v)$ proved in Proposition 3.6. Furthermore, this topology makes $\Phi_{1}(\cdot, v)$ lower semicontinuous and $\Phi_{1}(u, \cdot)$ is even continuous because $u$ does not occur explicitly in $\Phi_{1}$, cf. (10). To show the upper semicontinuity of the set-valued mapping $v \mapsto \operatorname{Argmin}_{\mathcal{U}} \Phi_{1}(\cdot, v)$, let us take a sequence $\left\{v_{k}\right\}$ converging weakly to $v$ and a sequence $\left\{u_{k}\right\}$ weakly convergent to $u$ and $u_{k} \in \operatorname{Argmin}_{\mathcal{U}} \Phi_{1}\left(\cdot, v_{k}\right)$, i.e.

$$
\Phi_{1}\left(u_{k}, v_{k}\right) \leq \Phi_{1}\left(\widetilde{u}, v_{k}\right)
$$

for all $\widetilde{u} \in \mathcal{U}$. Making a limit passage, by lower semicontinuity of $\Phi_{\ell}$ and upper semicontinuity of $\Phi_{\ell}(\widetilde{u}, \cdot)$, we get

$$
\Phi_{1}(u, v) \leq \liminf _{k \rightarrow \infty} \Phi_{1}\left(u_{k}, v_{k}\right) \leq \limsup _{k \rightarrow \infty} \Phi_{1}\left(\widetilde{u}, v_{k}\right) \leq \Phi_{1}(\widetilde{u}, v)
$$

which says that $u \in \operatorname{Argmin}_{\mathcal{U}} \Phi_{1}(\cdot, v)$. Hence, the upper semicontinuity of the mapping

$$
v \mapsto \operatorname{Argmin}_{\mathcal{U}} \Phi_{1}(\cdot, v)
$$

is shown. The case of $u \mapsto \operatorname{Argmin}_{\mathcal{V}} \Phi_{2}(u, \cdot)$ is analogous.

\section{Conceptual approximation scheme}

To implement the above game computationally, one needs to discretize the controlled system (1). This can be made by various ways, but one option is of a special interest: a suitable semi-explicit formula in time which would guarantee all previous estimates (i.e. stability and non-negativity of approximate solutions) and simultaneously would yield linear problems at each time level so that it allows for exact solutions to the discretized system. This is important especially in context of optimization where usage of iterative solvers would lead to inaccuracy also in the calculated gradient of $\Phi_{\ell}$ (i.e. in solution to the corresponding discrete adjoint problem) which would eventually make troubles in optimization routines which are usually very sensitive to any inaccuracy in the calculated gradient. To demonstrate the main features in an easy-to-observe way, we present only a conceptual discretization in time, keeping the spatial variable $x$ continuous; this semidiscretization in time is sometimes called Rothe's method if made in a fully implicit way (which is not our case, however).

We consider the time discretization step $\tau$, defined by $\tau=T / N$, where $N$ is a positive integer. Besides, we take some finite-dimensional subsets $\mathcal{U}_{h} \subset \mathcal{U}$ and $\mathcal{V}_{h} \subset \mathcal{V}$ where $h>0$ is a discretization parameter, assuming naturally that these sets are nested for $h \searrow 0$ and 
that $\bigcup_{h>0} \mathcal{U}_{h}$ and $\bigcup_{h>0} \mathcal{V}_{h}$ are $L^{r}(\Omega)$-dense in $\mathcal{U}$ and $\mathcal{V}$, respectively. Moreover, we assume these sets to be weakly closed; an example is a discretization of both $\mathcal{U}$ and $\mathcal{V}$ with piecewise constant controls on a finite-volume discretization of $\Omega$. We approximate then the Nash Equilibrium problem (10) by the following semi-discretized problem:

$$
\left\{\begin{array}{l}
\text { Find a Nash Equilibrium of } \\
J_{1, \tau}(y, z, u):=\frac{1}{r} \int_{\Omega} u^{r} \mathrm{~d} x+\tau \sum_{i=1}^{N} \frac{\beta_{11}}{2}\left\|y_{\tau}^{i}-y_{1, \mathrm{~d}, \tau}^{i}\right\|_{H^{\alpha}(\Omega)}^{2}+\frac{\beta_{21}}{2}\left\|z_{\tau}^{i}-z_{1, \mathrm{~d}, \tau}^{i}\right\|_{H^{\alpha}(\Omega)}^{2} \\
J_{2, \tau}(y, z, v):=\frac{1}{r} \int_{\Omega} v^{r} \mathrm{~d} x+\tau \sum_{i=1}^{N} \frac{\beta_{12}}{2}\left\|y_{\tau}^{i}-y_{2, \mathrm{~d}, \tau}^{i}\right\|_{H^{\alpha}(\Omega)}^{2}+\frac{\beta_{22}}{2}\left\|z_{\tau}^{i}-z_{2, \mathrm{~d}, \tau}^{i}\right\|_{H^{\alpha}(\Omega)}^{2} \\
\text { where }(u, v) \in \mathcal{U}_{h} \times \mathcal{V}_{h} \text { and } y=\left\{y_{\tau}^{i}\right\}_{i=1}^{N} \text { and } z=\left\{z_{\tau}^{i}\right\}_{i=1}^{N} \text { solve }(37)-(38):
\end{array}\right.
$$

$$
\begin{aligned}
& \begin{cases}\frac{y_{\tau}^{i}-y_{\tau}^{i-1}}{\tau}-d_{1} \Delta y_{\tau}^{i}=y_{\tau}^{i-1} a\left(1-\frac{y_{\tau}^{i}}{k}\right)-b \frac{y_{\tau}^{i} z_{\tau}^{i-1}}{1+e y_{\tau}^{i-1}} & \text { in } \Omega, \\
y_{\tau}^{i}=0 & \text { on } \Gamma,\end{cases} \\
& \begin{cases}\frac{z_{\tau}^{i}-z_{\tau}^{i-1}}{\tau}-d_{2} \Delta z_{\tau}^{i}=z^{i}\left(-c+d \frac{y_{\tau}^{i}}{1+e y_{\tau}^{i}}\right) & \text { in } \Omega, \\
z_{\tau}^{i}=0 & \text { on } \Gamma,\end{cases}
\end{aligned}
$$

for $i=1, \ldots, N$, while for $i=0$ we consider

$$
y^{0}=u, \quad z^{0}=v
$$

Here, in the general case, $y_{\ell, \mathrm{d}, \tau}^{i}:=\frac{1}{\tau} \int_{(i-1) \tau}^{i \tau} y_{\ell, \mathrm{d}}(t) \mathrm{d} t$ and similarly $z_{\ell, \mathrm{d}, \tau}^{i}:=\frac{1}{\tau} \int_{(i-1) \tau}^{i \tau} z_{\ell, \mathrm{d}}(t) \mathrm{d} t$. Let us remark that, if $y_{\ell, \mathrm{d}}, z_{\ell, \mathrm{d}} \in C\left([0, T] ; H^{\alpha}(\Omega)\right)$, we could take alternatively $y_{\ell, \mathrm{d}, \tau}^{i}=$ $y_{\ell, \mathrm{d}}(i \tau)$ and $z_{\ell, \mathrm{d}, \tau}^{i}=z_{\ell, \mathrm{d}}(i \tau)$. Let us denote by $y_{\tau} \in C\left(0, T ; L^{\infty}(\Omega)\right) \cap H_{0}^{1}(\Omega)$ the piecewise affine interpolation between $y^{0}, y^{1}, \ldots, y^{N}$, and by $\bar{y}_{\tau} \in L^{\infty}\left(0, T ; H_{0}^{1}(\Omega)\right)$ the piecewise constant interpolation of $y^{1}, y^{2}, \ldots, y^{N}$, i.e. $\bar{y}_{\tau}(t)=y_{\tau}^{i}$ for $t \in((i-1) \tau, i \tau)$ (the regularity for $y_{\tau}$ and $\bar{y}_{\tau}$ is proved in the Lemma below). The notation $z_{\tau}$ and $\bar{z}_{\tau}$ will have an analogous meaning.

Lemma 4.1. If $\tau<1 /\left(\frac{d k}{1+e k}-c\right)^{+}$, the elliptic problems in (37) have unique solutions $\left(y_{\tau}^{i}, z_{\tau}^{i}\right) \in\left(L^{\infty}(\Omega) \cap H_{0}^{1}(\Omega)\right)^{2}$ for all $i=1, \cdots, N$, which satisfy $0 \leq y_{\tau}^{i} \leq k$ and $0 \leq z_{\tau}^{i} \leq$ $\left(1+\tau\left(c-\frac{d k}{1+e k}\right)\right)^{-i} K$ provided $0 \leq u \leq k$ and $0 \leq v \leq K$.

Proof of Lemma 4.1. We use an induction argument. Let us suppose $y_{\tau}^{i-1}, z_{\tau}^{i-1} \in L^{\infty}(\Omega)$ are nonnegative and satisfy $0 \leq y_{\tau}^{i-1} \leq k$ and $0 \leq z_{\tau}^{i-1} \leq\left(1+\tau\left(c-\frac{d k}{1+e k}\right)\right)^{-(i-1)} K$. Then, we have to prove that $y_{\tau}^{i}, z_{\tau}^{i}$ satisfy the same hypothesis and also that $y_{\tau}^{i}, z_{\tau}^{i} \in H_{0}^{1}(\Omega)$.

Obviously, (37a) is a linear boundary-value problem for $y_{\tau}^{i}$ whose solution, in a weak sense, is defined by the integral identity

$$
\int_{\Omega}\left(\frac{1}{\tau}+a \frac{y_{\tau}^{i-1}}{k}+\frac{b z_{\tau}^{i-1}}{1+e y_{\tau}^{i-1}}\right) y_{\tau}^{i} \varphi+d_{1} \nabla y_{\tau}^{i} \cdot \nabla \varphi \mathrm{d} x=\int_{\Omega}\left(\frac{1}{\tau}+a\right) y_{\tau}^{i-1} \varphi \mathrm{d} x
$$

to be valid for all $\varphi \in H_{0}^{1}(\Omega)$. It is clear that, if $y_{\tau}^{i-1}, z_{\tau}^{i-1} \in L^{\infty}(\Omega)$ are non-negative, the left-hand side of (39) defines a continuous and positive bilinear form on $H_{0}^{1}(\Omega)$ and the right-hand side defines a continuous linear form on $H_{0}^{1}(\Omega)$. Therefore, by classical 
Lax-Milgram theorem, it possesses a unique solution $y_{\tau}^{i} \in H_{0}^{1}(\Omega)$. Let us see that $y_{\tau}^{i}$ is non-negative. Testing (39) by $\left(y_{\tau}^{i}\right)^{-}$one gets

$$
\frac{1}{\tau}\left\|\left(y_{\tau}^{i}\right)^{-}\right\|_{2}^{2}+d_{1}\left\|\nabla\left(y_{\tau}^{i}\right)^{-}\right\|_{2}^{2} \leq \int_{\Omega}\left(\frac{1}{\tau}+a\right) y_{\tau}^{i-1}\left(y_{\tau}^{i}\right)^{-} \mathrm{d} x \leq 0 .
$$

Hence, we get $\left(y_{\tau}^{i}\right)^{-}=0$ and therefore $y_{\tau}^{i} \geq 0$ a.e. in $\Omega$. Let us prove that $y_{\tau}^{i} \leq k$. To do it, we write (37a) in the following equivalent way:

$$
\begin{cases}\frac{\left(y_{\tau}^{i}-k\right)-\left(y_{\tau}^{i-1}-k\right)}{\tau}-d_{1} \Delta y_{\tau}^{i}=y_{\tau}^{i-1} a\left(1-\frac{y_{\tau}^{i}}{k}\right)-b \frac{y_{\tau}^{i} z_{\tau}^{i-1}}{1+e y_{\tau}^{i-1}} & \text { in } \Omega \\ y_{\tau}^{i}=0 & \text { on } \Gamma .\end{cases}
$$

Then, testing by $\left(y_{\tau}^{i}-k\right)^{+}$we get

$$
\begin{aligned}
\left\|\left(y_{\tau}^{i}-k\right)^{+}\right\|_{2}^{2} & +\tau d_{1}\left\|\nabla\left(y_{\tau}^{i}-k\right)^{+}\right\|_{2}^{2}=\int_{\Omega}\left[\left(y_{\tau}^{i-1}-k\right)\left(y_{\tau}^{i}-k\right)^{+}\right. \\
& \left.-\tau\left(a y_{\tau}^{i-1} \frac{y_{\tau}^{i}-k}{k}\left(y_{\tau}^{i}-k\right)^{+}+b \frac{y_{\tau}^{i}\left(y_{\tau}^{i}-k\right)^{+} z_{\tau}^{i-1}}{1+e y_{\tau}^{i-1}}\right)\right] \mathrm{d} x \leq 0
\end{aligned}
$$

since $0 \leq y_{\tau}^{i-1} \leq k, 0 \leq y_{\tau}^{i}$ and $z_{\tau}^{i-1} \geq 0$. Hence, $y_{\tau}^{i} \leq k$ a.e. in $\Omega$.

Now, (37b) is a linear boundary-value problem for $z_{\tau}^{i}$ whose solution, in a weak sense, is defined by the integral identity

$$
\int_{\Omega}\left(\frac{1}{\tau}+c-d \frac{y_{\tau}^{i}}{1+e y_{\tau}^{i}}\right) z_{\tau}^{i} \varphi+d_{2} \nabla z_{\tau}^{i} \cdot \nabla \varphi \mathrm{d} x=\int_{\Omega} \frac{z_{\tau}^{i-1}}{\tau} \varphi \mathrm{d} x
$$

to be valid for all $\varphi \in H_{0}^{1}(\Omega)$. Further, if $0 \leq y_{\tau}^{i-1} \leq k$ a.e. and $\tau<1 /\left(\frac{d k}{1+e k}-c\right)^{+}$ (this bound can be improved a little by using Poincaré's inequality), the left hand side of (42) defines a continuous and positive bilinear form on $H_{0}^{1}(\Omega)$. Therefore, by classical Lax-Milgram theorem, it possesses a unique solution $z_{\tau}^{i} \in H_{0}^{1}(\Omega)$. Let us see that $z_{\tau}^{i}$ is non-negative. Testing (42) by $\left(z_{\tau}^{i}\right)^{-}$one gets

$$
\left(\frac{1}{\tau}+c-d k\right)\left\|\left(z_{\tau}^{i}\right)^{-}\right\|_{2}^{2}+d_{2}\left\|\nabla\left(z_{\tau}^{i}\right)^{-}\right\|_{2}^{2} \leq \int_{\Omega} \frac{z_{\tau}^{i-1}}{\tau}\left(z_{\tau}^{i}\right)^{-} \mathrm{d} x \leq 0
$$

Hence, if $\tau<1 /\left(\frac{d k}{1+e k}-c\right)^{+}$, we get $\left(z_{\tau}^{i}\right)^{-}=0$ and therefore $z_{\tau}^{i} \geq 0$ a.e. in $\Omega$.

Finally, let us prove the upper bound for $z_{\tau}^{i}$. To do it, we look for an arbitrary constant $M>0$ such that $z_{\tau}^{i} \leq M$. We write $(37 \mathrm{~b})$ in the following equivalent way:

$$
\begin{cases}\frac{\left(z_{\tau}^{i}-M\right)-\left(z_{\tau}^{i-1}-M\right)}{\tau}-d_{2} \Delta z_{\tau}^{i}=z_{\tau}^{i}\left(-c+d \frac{y_{\tau}^{i}}{1+e y_{\tau}^{i}}\right) & \text { in } \Omega, \\ z_{\tau}^{i}=0 & \text { on } \Gamma .\end{cases}
$$

Then, testing by $\left(z_{\tau}^{i}-M\right)^{+}$we get

$$
\begin{aligned}
\left(\frac{1}{\tau}+c\right. & \left.-\frac{d k}{1+e k}\right)\left\|\left(z_{\tau}^{i}-M\right)^{+}\right\|_{2}^{2}+d_{2}\left\|\nabla\left(z_{\tau}^{i}-M\right)^{+}\right\|_{2}^{2} \\
& \leq \int_{\Omega}\left[\frac{1}{\tau}\left(z_{\tau}^{i-1}-M\right)+M\left(\frac{d k}{1+e k}-c\right)\right]\left(z_{\tau}^{i}-M\right)^{+} \mathrm{d} x .
\end{aligned}
$$

Let us see that we can choose $M$ such that

$$
\frac{1}{\tau}\left(z_{\tau}^{i-1}-M\right)+M\left(\frac{d k}{1+e k}-c\right) \leq 0 \quad \text { a.e.in } \Omega
$$


which is equivalent to

$$
M\left(\frac{1}{\tau}+c-\frac{d k}{1+e k}\right) \geq \frac{\left\|z_{\tau}^{i-1}\right\|_{\infty}}{\tau} .
$$

Now, a necessary condition for (45) is

$$
\frac{1}{\tau}>\left(\frac{d k}{1+e k}-c\right)^{+}
$$

which is true by hypothesis. Then, (45) is equivalent to

$$
M \geq \frac{\left\|z_{\tau}^{i-1}\right\|_{\infty}}{\tau(1 / \tau+c-d k /(1+e k))} .
$$

Under these conditions, we have $0 \leq z_{\tau}^{i} \leq M$ and therefore

$$
0 \leq z_{\tau}^{i} \leq\left(1+\tau\left(c-\frac{d k}{1+e k}\right)\right)^{-i} K
$$

Remark 4.2. The bound in Lemma 4.1 for $z_{\tau}^{i}$ is a discrete version of the one in Proposition 2.1 for $z(t)$, in the following way: Let us fix for instance $t=T$, so that $N \tau=T$. Then,

and

$$
z_{\tau}^{N} \leq\left(1+\tau\left(c-\frac{d k}{1+e k}\right)\right)^{-N} K
$$

$$
\lim _{N \rightarrow+\infty}\left(1+\tau\left(c-\frac{d k}{1+e k}\right)\right)^{-N}=\lim _{N \rightarrow+\infty}\left(1+\frac{T\left(c-\frac{d k}{1+e k}\right)}{N}\right)^{-N}=\mathrm{e}^{T\left(\frac{d k}{1+e k}-c\right)} .
$$

Lemma 4.3. If $\tau<1 /\left(\frac{d k}{1+e k}-c\right)^{+}$, the following a-priori estimates hold:

$$
\left\|\left(\bar{y}_{\tau}, \bar{z}_{\tau}\right)\right\|_{L^{\infty}(Q)^{2} \cap L^{2}\left(0, T ; H_{0}^{1}(\Omega)\right)^{2}} \leq C, \quad\left\|\left(\frac{\partial y_{\tau}}{\partial t}, \frac{\partial z_{\tau}}{\partial t}\right)\right\|_{L^{2}\left(0, T ; H^{-1}(\Omega)\right)^{2}} \leq C
$$

with $C>0$. Further, if $\tau \in\left(0,\left(\frac{d k}{1+e k}-c\right)^{+}-\varepsilon\right)$, with $\varepsilon>0$, then $C$ is independent of $\tau$. Proof of Lemma 4.3. Since $0 \leq y_{\tau}^{i} \leq k$ a.e. in $\Omega$, for $i=0, \cdots, N$, it is clear that $\left\|\bar{y}_{\tau}\right\|_{L^{\infty}(Q)} \leq C$, with $C$ independent of $\tau$. Furthermore,

$$
\begin{aligned}
z_{\tau}^{i} & \leq\left(1+\tau\left(c-\frac{d k}{1+e k}\right)\right)^{-i} K \\
& \leq\left\{\begin{array}{ll}
\left(1-\tau\left(\frac{d k}{1+e k}-c\right)\right)^{\frac{-T}{\tau}} K & \text { if } \frac{d k}{1+e k}-c \geq 0 \\
K & \text { if } \frac{d k}{1+e k}-c \leq 0
\end{array}\right\} \stackrel{\tau \rightarrow 0}{\longrightarrow} e^{T\left(\frac{d k}{1+e k}-c\right)^{+}} K,
\end{aligned}
$$

which implies that $\left\|\bar{z}_{\tau}\right\|_{L^{\infty}(Q)} \leq C$, with $C$ independent of $\tau$.

Now, testing (39) by $y_{\tau}^{i}$ and using Young's inequality we have

$$
\frac{1}{2 \tau}\left\|y_{\tau}^{i}\right\|_{2}^{2}+d_{1}\left\|\nabla y_{\tau}^{i}\right\|_{2}^{2} \leq \int_{\Omega}\left(\frac{1}{2 \tau}+\frac{a}{2}\right)\left(y_{\tau}^{i-1}\right)^{2}+\int_{\Omega} \frac{a}{2}\left(y_{\tau}^{i}\right)^{2} \mathrm{~d} x .
$$

Then, multiplying it by $\tau$ and summing for $i=1, \ldots, N$, we obtain

$$
\begin{aligned}
\frac{1}{2}\left\|y^{N}\right\|_{2}^{2}+d_{1}\left\|\bar{y}_{\tau}\right\|_{L^{2}\left(0, T ; H_{0}^{1}(\Omega)\right)}^{2} & \leq \frac{1}{2}\|u\|_{2}^{2}+\tau \frac{a}{2} \sum_{i=1}^{N} \int_{\Omega}\left(\left(y_{\tau}^{i-1}\right)^{2}+\left(y_{\tau}^{i}\right)^{2}\right) \mathrm{d} x \\
& \leq \frac{1}{2}\|u\|_{2}^{2}+T a k^{2}|\Omega|
\end{aligned}
$$


which implies that $\left\|\bar{y}_{\tau}\right\|_{L^{2}\left(0, T ; H_{0}^{1}(\Omega)\right)} \leq C$, with $C$ independent of $\tau$.

Now, testing (42) by $z_{\tau}^{i}$ and using Young inequality we have

$$
\begin{aligned}
\frac{1}{\tau}\left\|z_{\tau}^{i}\right\|_{2}^{2}+d_{2}\left\|\nabla z_{\tau}^{i}\right\|_{2}^{2} & \leq \int_{\Omega}\left(\frac{z_{\tau}^{i-1}}{\tau} z_{\tau}^{i}+\left(-c+d \frac{y_{\tau}^{i}}{1+e y_{\tau}^{i}}\right)\left(z_{\tau}^{i}\right)^{2}\right) \mathrm{d} x \\
& \leq \frac{1}{2 \tau}\left\|z_{\tau}^{i-1}\right\|_{2}^{2}+\frac{1}{2 \tau}\left\|z_{\tau}^{i-1}\right\|_{2}^{2}+\left(\frac{d k}{1+e k}-c\right)\left\|z_{\tau}^{i}\right\|_{2}^{2}
\end{aligned}
$$

and

$$
\frac{1}{2 \tau}\left\|z_{\tau}^{i}\right\|_{2}^{2}+d_{2}\left\|\nabla z_{\tau}^{i}\right\|_{2}^{2} \leq \frac{1}{2 \tau}\left\|z_{\tau}^{i-1}\right\|_{2}^{2}+\left(\frac{d k}{1+e k}-c\right)\left\|z_{\tau}^{i}\right\|_{2}^{2}
$$

Then, multiplying by $\tau$ and summing for $i=1, \ldots, N$, we obtain

$$
\frac{1}{2}\left\|z^{N}\right\|_{2}^{2}+d_{2}\left\|\bar{z}_{\tau}\right\|_{L^{2}\left(0, T ; H_{0}^{1}(\Omega)\right)}^{2} \leq \frac{1}{2}\|v\|_{2}^{2}+\left(\frac{d k}{1+e k}-c\right)\left\|\bar{z}_{\tau}\right\|_{L^{2}(Q)}^{2},
$$

which implies that $\left\|\bar{z}_{\tau}\right\|_{L^{2}\left(0, T ; H_{0}^{1}(\Omega)\right)} \leq C$, with $C$ independent of $\tau$. Now,

$$
\begin{aligned}
\left\|\frac{\partial y_{\tau}}{\partial t}\right\|_{L^{2}\left(0, T ; H^{-1}(\Omega)\right)}^{2} & =\int_{0}^{T} \sup _{\|\varphi\|_{H_{0}^{1}(\Omega)}=1}\left(\left\langle\frac{\partial y_{\tau}}{\partial t}, \varphi\right\rangle_{H^{-1}(\Omega) \times H_{0}^{1}(\Omega)}\right)^{2} \mathrm{~d} t \\
& =\tau \sum_{i=1}^{N} \sup _{\|\varphi\|_{H_{0}^{1}(\Omega)}}\left(\int_{\Omega} \frac{y_{\tau}^{i}-y_{\tau}^{i-1}}{\tau} \varphi \mathrm{d} x\right)^{2} .
\end{aligned}
$$

Then, from (39), we have that

$$
\begin{aligned}
\left\|\frac{\partial y_{\tau}}{\partial t}\right\|_{L^{2}\left(0, T ; H^{-1}(\Omega)\right)}^{2}= & \tau \sum_{i=1}^{N} \sup _{\|\varphi\|_{H_{0}^{1}(\Omega)}=1}\left[\int_{\Omega}-d_{1} \nabla y_{\tau}^{i} \cdot \nabla \varphi\right. \\
& \left.+\left(a y_{\tau}^{i-1}\left(1-\frac{y_{\tau}^{i}}{k}\right)-b \frac{y_{\tau}^{i} z_{\tau}^{i-1}}{1+e y_{\tau}^{i-1}}\right) \varphi \mathrm{d} x\right]^{2} \\
\leq & 3\left[d_{1}^{2}\left\|\bar{y}_{\tau}\right\|_{L^{2}\left(0, T ; H_{0}^{1}(\Omega)\right)}^{2}+a^{2} k^{2} T|\Omega|+b^{2} k^{2}\left\|_{\bar{z}_{\tau}}\right\|_{L^{\infty}(Q)}^{2} T|\Omega|\right],
\end{aligned}
$$

which implies that $\left\|y_{\tau}\right\|_{L^{2}\left(0, T ; H^{-1}(\Omega)\right)} \leq C$, with $C$ independent of $\tau$.

Finally, from (42), we have that

$$
\begin{aligned}
\left\|\frac{\partial z_{\tau}}{\partial t}\right\|_{L^{2}\left(0, T ; H^{-1}(\Omega)\right)}^{2} & =\tau \sum_{i=1}^{N} \sup _{\|\varphi\|_{H_{0}^{1}(\Omega)}=1}\left(\int_{\Omega} \frac{z_{\tau}^{i}-z_{\tau}^{i-1}}{\tau} \varphi \mathrm{d} x\right)^{2} \\
& =\tau \sum_{i=1}^{N} \sup _{\|\varphi\|_{H_{0}^{1}(\Omega)}=1}\left[\int_{\Omega}-d_{2} \nabla z_{\tau}^{i} \cdot \nabla \varphi-z_{\tau}^{i}\left(c-d \frac{y_{\tau}^{i}}{1+e y_{\tau}^{i}}\right) \varphi \mathrm{d} x\right]^{2} \\
& \leq 2 d_{2}^{2}\left\|\bar{z}_{\tau}\right\|_{L^{2}\left(0, T ; H_{0}^{1}(\Omega)\right)}^{2}+2\left(c+\frac{d k}{1+e k}\right)^{2}\left\|\bar{z}_{\tau}\right\|_{L^{\infty}(Q)}^{2} T|\Omega|,
\end{aligned}
$$

which implies that $\left\|z_{\tau}\right\|_{L^{2}\left(0, T ; H^{-1}(\Omega)\right)} \leq C$, with $C$ independent of $\tau$.

For further purposes, we need also to involve a continuous dependence on the initial conditions. This is, however, just a simple extension of the convergence proof. For this purpose, let us consider another parameter $h>0$ and sequences $\left\{u_{h}\right\}_{h>0} \subset \mathcal{U}$ with $u_{h} \in \mathcal{U}_{h}$ and $\left\{v_{h}\right\}_{h>0} \subset \mathcal{V}$ with $v_{h} \in \mathcal{V}_{h}$ converging for $h \searrow 0$ weakly in $L^{r}(\Omega)$ to $u$ and $v$, 
respectively, and denote by $\left(y_{\tau, h}, z_{\tau, h}\right)$ solution to the approximate system (37) with the initial conditions $y^{0}=u_{h}$ and $z^{0}=v_{h}$ instead of (38).

Lemma 4.4. For $\tau, h \searrow 0, \bar{y}_{\tau, h} \rightarrow y$ and $\bar{z}_{\tau, h} \rightarrow z$ strongly in $L^{2}\left(0, T ; H^{\alpha}(\Omega)\right)$, and $(y, z)$ solves (1).

Proof. There is a "technical" difficulty that $y_{\tau, h}, z_{\tau, h} \notin L^{2}\left(0, T ; H_{0}^{1}(\Omega)\right)$ if $u_{h}, v_{h} \in L^{2}(\Omega) \backslash$ $H_{0}^{1}(\Omega)$. To overcome it, let us introduce the auxiliary function $\widehat{y}_{\tau, h} \in C\left(0, T ; H_{0}^{1}(\Omega)\right)$ which is constant on $[0, \tau]$ and coincides with $y_{\tau, h}$ on $[\tau, T]$. The analogous meaning has $\widehat{z}_{\tau, h}$. The bounds obtained in the proof of Lemma 4.3 say that the sequence $\left\{\left(\widehat{y}_{\tau, h}, \widehat{z}_{\tau, h}\right)\right\}$ is bounded in $\mathcal{W}^{2}$ for $\tau>0$ small enough.

We will need also the "retarded" function $\bar{y}_{\tau, h}(.-\tau)$, denoted by $\bar{y}_{\tau, h}^{\mathrm{R}}$; of course, we put naturally $\bar{y}_{\tau, h}^{\mathrm{R}}(t):=u_{h}$ for $t<\tau$. The function $\bar{z}_{\tau, h}^{\mathrm{R}}$ is defined analogously.

We can choose a subsequence such that $\left(\widehat{y}_{\tau, h}, \widehat{z}_{\tau, h}\right) \rightarrow(\widehat{y}, \widehat{z})$ weakly in $\mathcal{W}^{2}$, and also $\left(y_{\tau, h}, z_{\tau, h}\right) \rightarrow(y, z)$ weakly in $L^{2}(Q)^{2}$, and $\left(\bar{y}_{\tau, h}, \bar{z}_{\tau, h}\right) \rightarrow(\bar{y}, \bar{z})$ weakly in $L^{2}\left(0, T ; H_{0}^{1}(\Omega)\right)^{2}$, and $\left(\bar{y}_{\tau, h}^{\mathrm{R}}, \bar{z}_{\tau, h}^{\mathrm{R}}\right) \rightarrow\left(\bar{y}^{\mathrm{R}}, \bar{z}^{\mathrm{R}}\right)$ weakly in $L^{2}(Q)^{2}$.

Due to $\left\|\widehat{y}_{\tau, h}-y_{\tau, h}\right\|_{L^{2}(Q)}=\sqrt{\tau / 3}\left\|u_{h}-y_{\tau, h}^{1}\right\|_{L^{2}(\Omega)}=\mathcal{O}(\sqrt{\tau})$, we can claim that $\widehat{y}=y$. By a similar argument, also $\widehat{z}=z$. As $\left\|\bar{y}_{\tau, h}-y_{\tau, h}\right\|_{L^{2}\left(0, T ; H^{-1}(\Omega)\right)}=\sqrt{\tau / 3}\left\|\frac{\partial}{\partial t} y_{\tau, h}\right\|_{L^{2}\left(0, T ; H^{-1}(\Omega)\right)}=$ $\mathcal{O}(\sqrt{\tau})$, we have also $\bar{y}=y$. Similarly also $\bar{z}=z$. Likewise, $\left\|\bar{y}_{\tau, h}-\bar{y}_{\tau, h}^{\mathrm{R}}\right\|_{L^{2}\left(0, T ; H^{-1}(\Omega)\right)}=$ $\sqrt{\tau}\left\|\frac{\partial}{\partial t} y_{\tau, h}\right\|_{L^{2}\left(0, T ; H^{-1}(\Omega)\right)}=\mathcal{O}(\sqrt{\tau})$, we have also $\bar{y}^{\mathrm{R}}=\bar{y}=y$, and similarly also $\bar{z}^{\mathrm{R}}=z$.

Because of the compact embedding $H_{0}^{1}(\Omega) \subset H^{\alpha}(\Omega)$, by Aubin-Lions's theorem, the convergence $\left(\widehat{y}_{\tau, h}, \widehat{z}_{\tau, h}\right) \rightarrow(y, z)$ is strong in $L^{2}\left(0, T ; H^{\alpha}(\Omega)\right)^{2}$. As $\left\|\widehat{y}_{\tau, h}-\bar{y}_{\tau, h}\right\|_{L^{2}\left(0, T ; H_{0}^{1}(\Omega)\right)}$ is obviously bounded and

$$
\left\|\widehat{y}_{\tau, h}-\bar{y}_{\tau, h}\right\|_{L^{2}\left(0, T ; H^{-1}(\Omega)\right)}^{2}=\frac{\tau}{3}\left\|\frac{\partial y_{\tau, h}}{\partial t}\right\|_{L^{2}\left(\tau, T ; H^{-1}(\Omega)\right)}^{2} \leq \frac{\tau}{3}\left\|\frac{\partial y_{\tau, h}}{\partial t}\right\|_{L^{2}\left(0, T ; H^{-1}(\Omega)\right)}^{2} \leq \frac{\tau C}{3}
$$

with $C$ from (47), and as $H^{\alpha}(\Omega)$ is an interpolation space between $H_{0}^{1}(\Omega)$ and $H^{-1}(\Omega)$ in the sense that, for all $v \in H^{\alpha}(\Omega)$,

$$
\|v\|_{H^{\alpha}(\Omega)} \leq C\|v\|_{H_{0}^{1}(\Omega)}^{(1+\alpha) / 2}\|v\|_{H^{-1}(\Omega)}^{(1-\alpha) / 2}
$$

for some $C$, we obtain

$$
\left\|\widehat{y}_{\tau, h}-\bar{y}_{\tau, h}\right\|_{L^{2}\left(0, T ; H^{\alpha}(\Omega)\right)}=\mathcal{O}\left(\tau^{(1-\alpha) / 4}\right),
$$

from which one can claim that also $\bar{y}_{\tau, h} \rightarrow y$ strongly in $L^{2}\left(0, T ; H^{\alpha}(\Omega)\right)$. By analogous arguments, also $\bar{z}_{\tau, h} \rightarrow z$ strongly in $L^{2}\left(0, T ; H^{\alpha}(\Omega)\right)$.

Now we are to prove that $(y, z)$ solves $(1)$. In terms of the introduced functions $y_{\tau, h}$, $\bar{y}_{\tau, h}, \bar{y}_{\tau, h}^{\mathrm{R}}, z_{\tau, h}, \bar{z}_{\tau, h}$, and $\bar{z}_{\tau, h}^{\mathrm{R}}$, the scheme (37) can be written down in a "compact" form as

$$
\begin{array}{ll}
\frac{\partial y_{\tau, h}}{\partial t}-d_{1} \Delta \bar{y}_{\tau, h}=a \bar{y}_{\tau, h}^{\mathrm{R}}\left(1-\frac{\bar{y}_{\tau, h}}{k}\right)-b \frac{\bar{y}_{\tau, h} \bar{z}_{\tau, h}^{\mathrm{R}}}{1+e \bar{y}_{\tau, h}^{\mathrm{R}}} & \text { on } Q, \\
\frac{\partial z_{\tau, h}}{\partial t}-d_{2} \Delta \bar{z}_{\tau, h}=-c \bar{z}_{\tau, h}+d \frac{\bar{y}_{\tau, h} \bar{z}_{\tau, h}}{1+e \bar{y}_{\tau, h}} & \text { on } Q, \\
\bar{y}_{\tau, h}=0, & \text { on } \Sigma, \\
y_{\tau, h}(0, \cdot)=u_{h}, \quad \bar{z}_{\tau, h}=0 & \text { on } \Omega .
\end{array}
$$


We already know that $\left(\bar{y}_{\tau, h}, \bar{z}_{\tau, h}\right) \rightarrow(y, z)$ strongly in $L^{2}(Q)$. Like $(48)$, we have also

$$
\left\|\bar{y}_{\tau, h}-\bar{y}_{\tau, h}^{\mathrm{R}}\right\|_{L^{2}\left(\tau, T ; H^{-1}(\Omega)\right)}^{2}=\tau\left\|\frac{\partial y_{\tau, h}}{\partial t}\right\|_{L^{2}\left(\tau, T ; H^{-1}(\Omega)\right)}^{2} \leq \tau\left\|\frac{\partial y_{\tau, h}}{\partial t}\right\|_{L^{2}\left(0, T ; H^{-1}(\Omega)\right)}^{2} \leq \tau C
$$

and also $\left\|\bar{y}_{\tau, h}-\bar{y}_{\tau, h}^{\mathrm{R}}\right\|_{L^{2}\left(\tau, T ; H_{0}^{1}(\Omega)\right)} \leq 2 C$ with $C$ from $(47)$; note that the last estimate can not hold in $L^{2}\left(0, T ; H_{0}^{1}(\Omega)\right)$ if $u_{h} \in L^{2}(\Omega) \backslash H_{0}^{1}(\Omega)$. Using the fact that $L^{2}(\Omega)$ is interpolant between $H_{0}^{1}(\Omega)$ and $H^{-1}(\Omega)$, cf. (49) for $\alpha=0$, we obtain $\left\|\bar{y}_{\tau, h}-\bar{y}_{\tau, h}^{\mathrm{R}}\right\|_{L^{2}\left(\tau, T ; L^{2}(\Omega)\right)}=\mathcal{O}(\sqrt{\tau})$ so that

$$
\left\|\bar{y}_{\tau, h}-\bar{y}_{\tau, h}^{\mathrm{R}}\right\|_{L^{2}(Q)}^{2}=\tau\left\|y_{\tau, h}^{1}-u\right\|_{L^{2}(\Omega)}^{2}+\left\|\bar{y}_{\tau, h}-\bar{y}_{\tau, h}^{\mathrm{R}}\right\|_{L^{2}\left(\tau, T ; L^{2}(\Omega)\right)}^{2}=\mathcal{O}(\tau)
$$

because obviously $\left\|u_{h}-y_{\tau, h}^{1}\right\|_{L^{2}(\Omega)} \leq 2 C$ with $C$ from (47). Therefore, we can claim that also $\bar{y}_{\tau, h}^{\mathrm{R}} \rightarrow y$ strongly in $L^{2}(Q)$. By analogous arguments, also $\bar{z}_{\tau, h}^{\mathrm{R}} \rightarrow z$ strongly in $L^{2}(Q)$.

This allows us already to make limit passage in (51). Note that the nonlinearity $\mathbb{R}_{+}^{2} \times$ $\mathbb{R}^{2} \rightarrow \mathbb{R}^{2}:\left(y, y^{\prime}, z, z^{\prime}\right) \mapsto\left(a y^{\prime}(1-y / k)-b y z^{\prime} /\left(1+e y^{\prime}\right),-c z+d y z /(1+e y)\right)$ has at most quadratic growth so that, by continuity of the respective Nemytskil mapping $L^{2}(Q)^{4} \rightarrow$ $L^{1}(Q)^{2}$ if restricted to nonnegative arguments $y$ 's, the right-hand sides of $(51 \mathrm{a}, \mathrm{b})$ converge strongly in $L^{1}(Q)$ to $a y(1-y / k)-b y z /(1+e y)$ and $-c z+d y z /(1+e y)$, respectively. The limit passage in the left-hand-side terms in $(51 \mathrm{a}, \mathrm{b})$ is obvious because they are linear. Note that the mapping $y \mapsto y(0, \cdot): \mathcal{W} \rightarrow L^{2}(\Omega)$ is (weak, weak)-continuous which allows to pass to the limit in the initial conditions $(51 \mathrm{~d})$.

Due to uniqueness of the weak solution to $(1)$, the whole sequence $\left\{\left(\bar{y}_{\tau, h}, \bar{z}_{\tau, h}\right)\right\}_{\tau>0}$ converges.

We could now do the analysis of convexity analogous to Proposition 3.6 for the discrete game (36). This would incorporate only a lot of work but not new ideas. Therefore, we will rather base further considerations only on convexity of the continuous problem. To do that, the continuous game (10) can be exploited in some extent. Let us still introduce the notation $\bar{y}_{\ell, \mathrm{d}, \tau}(t, x)=y_{\ell, \mathrm{d}, \tau}^{i}(x)$ for $(i-1) \tau<t<i \tau, \bar{z}_{\ell, \mathrm{d}, \tau}$ is defined analogously, and

$$
\Phi_{1, \tau}(u, v):=J_{1, \tau}\left(\bar{y}_{\tau}, \bar{z}_{\tau}, u\right), \quad \Phi_{2, \tau}(u, v):=J_{2, \tau}\left(\bar{y}_{\tau}, \bar{z}_{\tau}, v\right),
$$

where $\left(\bar{y}_{\tau}, \bar{z}_{\tau}\right)=\left(\bar{y}_{\tau}, \bar{z}_{\tau}\right)(u, v)$ is the solution to (37) and where $J_{1, \tau}$ and $J_{2, \tau}$ is just as $J_{1}$ and $J_{2}$ but with $y_{\ell, \mathrm{d}, \tau}$ and $z_{\ell, \mathrm{d}, \tau}$ instead of $y_{\ell, \mathrm{d}}$ and $z_{\ell, \mathrm{d}}$, respectively, i.e.

$$
\begin{aligned}
& J_{1, \tau}(y, z, u)=\frac{1}{r}\|u\|_{L^{r}(\Omega)}^{r}+\frac{\beta_{11}}{2}\left\|y-y_{1, \mathrm{~d}, \tau}\right\|_{L^{2}\left(0, T ; H^{\alpha}(\Omega)\right)}^{2}+\frac{\beta_{21}}{2}\left\|z-z_{1, \mathrm{~d}, \tau}\right\|_{L^{2}\left(0, T ; H^{\alpha}(\Omega)\right)}^{2} \\
& J_{2, \tau}(y, z, v)=\frac{1}{r}\|v\|_{L^{r}(\Omega)}^{r}+\frac{\beta_{12}}{2}\left\|y-y_{2, \mathrm{~d}, \tau}\right\|_{L^{2}\left(0, T ; H^{\alpha}(\Omega)\right)}^{2}+\frac{\beta_{22}}{2}\left\|z-z_{2, \mathrm{~d}, \tau}\right\|_{L^{2}\left(0, T ; H^{\alpha}(\Omega)\right)}^{2} .
\end{aligned}
$$

We point out that these functionals are the same as those defined in (36).

For a tolerance $\varepsilon>0$ we say that a couple $\left(u^{*}, v^{*}\right)$ is an $\varepsilon$-solution of the approximate game if

$$
\begin{aligned}
\left(u^{*}, v^{*}\right) \in \mathcal{U}_{h} \times \mathcal{V}_{h}: \quad \forall u \in \mathcal{U}_{h}: & \Phi_{1, \tau}\left(u^{*}, v^{*}\right) \leq \Phi_{1, \tau}\left(u, v^{*}\right)+\varepsilon \\
& \forall v \in \mathcal{V}_{h}: \quad \Phi_{2, \tau}\left(u^{*}, v^{*}\right) \leq \Phi_{2, \tau}\left(u^{*}, v\right)+\varepsilon
\end{aligned}
$$

Obviously, existence of such an $\varepsilon$-solution is trivial if $\varepsilon$ is sufficiently large but then the relevance with the original game is rather lost. Hence, it is important to have a chance to push $\varepsilon$ to zero: 
Proposition 4.5. There is a function $E=E(\tau, h)>0$ such that $\lim _{\tau \rightarrow 0, h \rightarrow 0} E(\tau, h)=0$ and, for any $\tau>0, h>0, \varepsilon \geq E(\tau, h)$, there is an $\varepsilon$-solution $\left(u_{\tau, h, \varepsilon}^{*}, v_{\tau, h, \varepsilon}^{*}\right)$ to $(56)$. Moreover, any sequence of such $\varepsilon$-solutions obtained by pushing $(\tau, h, \varepsilon)$ to zero under the "stability condition" $\varepsilon \geq E(\tau, h)$ contains an $L^{r}(\Omega)^{2}$-weakly convergent subsequence, i.e. (in terms of the same indices),

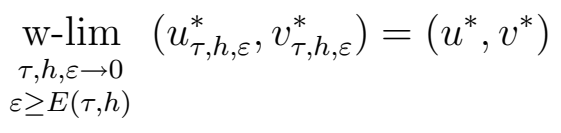

and any $\left(u^{*}, v^{*}\right)$ obtained by such way solves the original game (56).

Proof. The first terms in (54)-(55) are uniformly continuous on $\mathcal{U}$ and on $\mathcal{V}$. Let us denote the remaining terms in (54)-(55) by $\Phi_{1, \tau}^{0}(u, v):=\Phi_{1, \tau}(u, v)-\frac{1}{r}\|u\|_{L^{r}(\Omega)}^{r}$ and $\Phi_{2, \tau}^{0}(u, v):=$ $\Phi_{2, \tau}(u, v)-\frac{1}{r}\|v\|_{L^{r}(\Omega)}^{r}$. Similarly, we put $\Phi_{1}^{0}(u, v):=\Phi_{1}(u, v)-\frac{1}{r}\|u\|_{L^{r}(\Omega)}^{r}$ and $\Phi_{2}^{0}(u, v):=$ $\Phi_{2}(u, v)-\frac{1}{r}\|v\|_{L^{r}(\Omega)}^{r}$.

We proved before that the functionals $\Phi_{\ell}^{0}$ are $L^{r}(\Omega)$-weakly continuous on the compact set $\mathcal{U} \times \mathcal{V}$, hence they are uniformly continuous. Then they remain uniformly continuous in the $L^{r}(\Omega)$-strong uniformity; let $m_{\ell}$ denote the modulus of continuity in the sense that $\left|\Phi_{1}(u, v)-\Phi_{1}(\widetilde{u}, \widetilde{v})\right| \leq\left|\Phi_{1}^{0}(u, v)-\Phi_{1}^{0}(\widetilde{u}, \widetilde{v})\right|+\frac{1}{r}\|u-\widetilde{u}\|_{L^{r}(\Omega)}^{r} \leq m_{1}\left(\|u-\widetilde{u}\|_{L^{r}(\Omega)}+\|v-\widetilde{v}\|_{L^{r}(\Omega)}\right)$, and similar estimate holds for $\Phi_{2}$, too.

Let $\mathcal{E}_{\ell}(\tau):=\max _{(u, v) \in \mathcal{U} \times \mathcal{V}}\left|\Phi_{\ell, \tau}(u, v)-\Phi_{\ell}(u, v)\right|=\max _{(u, v) \in \mathcal{U} \times \mathcal{V}}\left|\Phi_{\ell, \tau}^{0}(u, v)-\Phi_{\ell}^{0}(u, v)\right|$. By Lemma 4.4, we have $\lim _{\tau \rightarrow 0} \mathcal{E}_{\ell}(\tau)=0$.

Let us still denote $\mathcal{E}_{h}^{\mathcal{U}}=\max _{u \in \mathcal{U}} \min _{\widetilde{u} \in \mathcal{U}_{h}}\|u-\widetilde{u}\|_{L^{r}(\Omega)}$, and analogous meaning has $\mathcal{E}_{h}^{\mathcal{V}}$. Our approximation assumption ensures $\lim _{h \rightarrow 0} \mathcal{E}_{h}^{\mathcal{U}}=0$ and $\lim _{h \rightarrow 0} \mathcal{E}_{h}^{\mathcal{V}}=0$.

Let us consider a solution $\left(u^{*}, v^{*}\right)$ to the original game (56) and take $\left(u_{h}, v_{h}\right) \in \mathcal{U}_{h} \times \mathcal{V}_{h}$ such that $\left\|u_{h}-u^{*}\right\|_{L^{r}(\Omega)} \leq \mathcal{E}_{h}^{\mathcal{U}}$ and $\left\|v_{h}-v^{*}\right\|_{L^{r}(\Omega)} \leq \mathcal{E}_{h}^{\mathcal{V}}$.

Then, for all $u \in \mathcal{U}$, it holds

$$
\begin{aligned}
\Phi_{1, \tau}\left(u_{h}, v_{h}\right) \leq & \Phi_{1}\left(u_{h}, v_{h}\right)+\mathcal{E}_{1}(\tau) \\
\leq & \Phi_{1}\left(u^{*}, v^{*}\right)+\mathcal{E}_{1}(\tau)+m_{1}\left(\left\|u_{h}-u^{*}\right\|_{L^{r}(\Omega)}+\left\|v_{h}-v^{*}\right\|_{L^{r}(\Omega)}\right) \\
\leq & \Phi_{1}\left(u, v^{*}\right)+\mathcal{E}_{1}(\tau)+m_{1}\left(\left\|u_{h}-u^{*}\right\|_{L^{r}(\Omega)}+\left\|v_{h}-v^{*}\right\|_{L^{r}(\Omega)}\right) \\
\leq & \Phi_{1}\left(u, v_{h}\right)+\mathcal{E}_{1}(\tau)+m_{1}\left(\left\|u_{h}-u^{*}\right\|_{L^{r}(\Omega)}+\left\|v_{h}-v^{*}\right\|_{L^{r}(\Omega)}\right) \\
& \quad+m_{1}\left(\left\|v_{h}-v^{*}\right\|_{L^{r}(\Omega)}\right) \\
\leq & \Phi_{1, \tau}\left(u, v_{h}\right)+2 \mathcal{E}_{1}(\tau)+2 m_{1}\left(\left\|u_{h}-u^{*}\right\|_{L^{r}(\Omega)}+\left\|v_{h}-v^{*}\right\|_{L^{r}(\Omega)}\right) \\
& +m_{1}\left(\left\|v_{h}-v^{*}\right\|_{L^{r}(\Omega)}\right) \\
\leq & \Phi_{1, \tau}\left(u, v_{h}\right)+2 \mathcal{E}_{1}(\tau)+2 m_{1}\left(\mathcal{E}_{h}^{\mathcal{U}}+\mathcal{E}_{h}^{\mathcal{V}}\right) .
\end{aligned}
$$

Similarly, one can get $\Phi_{2, \tau}\left(u_{h}, v_{h}\right) \leq \Phi_{2, \tau}\left(u_{h}, v\right)+2 \mathcal{E}_{2}(\tau)+2 m_{2}\left(\mathcal{E}_{h}^{\mathcal{U}}+\mathcal{E}_{h}^{\mathcal{V}}\right)$ for any $v \in \mathcal{V}$. Thus, in particular, considering $(u, v) \in \mathcal{U}_{h} \times \mathcal{V}_{h}$ only, we can see that $\left(u_{h}, v_{h}\right)$ is an $\varepsilon$-solution to the approximate game (56) for

$$
\varepsilon \geq E(\tau, h):=2 \max _{\ell=1,2}\left(\mathcal{E}_{\ell}(\tau)+m_{\ell}\left(\mathcal{E}_{h}^{\mathcal{U}}+\mathcal{E}_{h}^{\mathcal{V}}\right)\right) .
$$

Furthermore, let us take $\left(u_{\tau, h, \varepsilon}^{*}, v_{\tau, h, \varepsilon}^{*}\right) \in \mathcal{U}_{h} \times \mathcal{V}_{h}$ with the above specified properties, i.e. $\Phi_{1, \tau}\left(u_{\tau, h, \varepsilon}^{*}, v_{\tau, h, \varepsilon}^{*}\right) \leq \Phi_{1, \tau}\left(u, v_{\tau, h, \varepsilon}^{*}\right)+\varepsilon$ and $\Phi_{2, \tau}\left(u_{\tau, h, \varepsilon}^{*}, v_{\tau, h, \varepsilon}^{*}\right) \leq \Phi_{2, \tau}\left(u_{\tau, h, \varepsilon}^{*}, v\right)+\varepsilon$ for all 
$(u, v) \in \mathcal{U}_{h} \times \mathcal{V}_{h}$. Passing to the limit with $(\tau, h, \varepsilon) \rightarrow 0$, having in mind a subsequence satisfying (57) we obtain

$$
\Phi_{1}\left(u^{*}, v^{*}\right) \leq \Phi_{1}\left(u, v^{*}\right) \quad \text { and } \quad \Phi_{2}\left(u^{*}, v^{*}\right) \leq \Phi_{2}\left(u^{*}, v\right)
$$

for any $(u, v) \in \mathcal{U}_{h} \times \mathcal{V}_{h}$ with $h>0$ arbitrary. Note that we employed also $y_{\ell, \mathrm{d}, \tau} \rightarrow y_{\ell, \mathrm{d}}$ and $z_{\ell, \mathrm{d}, \tau} \rightarrow z_{\ell, \mathrm{d}}$ in the norm of $L^{2}\left(0, T ; H^{\alpha}(\Omega)\right)$, and the weak lower semicontinuity of $\Phi_{\ell}$ and also the strong continuity of $\Phi_{\ell}$. By the strong continuity of both $\Phi_{1}$ and $\Phi_{2}$, (59) holds for any $(u, v) \in \mathcal{U} \times \mathcal{V}$.

For simplicity of notation, from now on we denote $y^{i}:=y_{\tau}^{i}, z^{i}:=z_{\tau}^{i}, y_{l, \mathrm{~d}}^{i}:=y_{l, \mathrm{~d}, \tau}^{i}$ and $z_{l, \mathrm{~d}}^{i}:=z_{l, \mathrm{~d}, \tau}^{i}$. Now, for the time discretized problem, the Lagrange function for the $\ell$-th player is the function

$$
L_{\ell, \tau}:\left(H_{0}^{1}(\Omega)^{N} \cap L^{\infty}(\Omega)^{N}\right)^{2} \times \mathcal{U} \times \mathcal{V} \times\left(H_{0}^{1}(\Omega)^{N}\right)^{2} \longrightarrow \mathbb{R}
$$

defined, for $y \geq 0$, by

$$
\begin{aligned}
L_{\ell, \tau}(y, z, u, v, p, q) & :=\int_{\Omega}\left((2-\ell) \frac{1}{r} u^{r}+(\ell-1) \frac{1}{r} v^{r}\right) \mathrm{d} x \\
& +\frac{\tau}{2} \sum_{i=1}^{N}\left(\beta_{1 \ell}\left\|y^{i}-y_{\ell, d}^{i}\right\|_{H^{\alpha}(\Omega)}^{2}+\beta_{2 \ell}\left\|z^{i}-z_{\ell, d}^{i}\right\|_{H^{\alpha}(\Omega)}^{2}\right) \\
& -\tau \int_{\Omega} p^{1}\left(\frac{y^{1}-u}{\tau}-d_{1} \Delta y^{1}-u a\left(1-\frac{y^{1}}{k}\right)+b \frac{y^{1} v}{1+e u}\right) \mathrm{d} x \\
& -\tau \sum_{i=2}^{N} \int_{\Omega} p^{i}\left(\frac{y^{i}-y^{i-1}}{\tau}-d_{1} \Delta y^{i}-y^{i-1} a\left(1-\frac{y^{i}}{k}\right)+b \frac{y^{i} z^{i-1}}{1+e y^{i-1}}\right) \mathrm{d} x \\
& -\tau \int_{\Omega} q^{1}\left(\frac{z^{1}-v}{\tau}-d_{2} \Delta z^{1}-z^{1}\left(-c+d \frac{y^{1}}{1+e y^{1}}\right)\right) \mathrm{d} x \\
& -\tau \sum_{i=2}^{N} \int_{\Omega} q^{i}\left(\frac{z^{i}-z^{i-1}}{\tau}-d_{2} \Delta z^{i}-z^{i}\left(-c+d \frac{y^{i}}{1+e y^{i}}\right)\right) \mathrm{d} x .
\end{aligned}
$$

We point out that here the integrals are denoting the duality pair $\langle\cdot, \cdot\rangle_{H_{0}^{1}(\Omega) \times H^{-1}(\Omega)}$. The adjoint system for the $\ell$-th player is $\left[L_{\ell, \tau}\right]_{(y, z)}^{\prime}(y, z, u, v, p, q)=0$. Let us denote $p=p_{l}$ and $q=q_{l}$ to the solution of $\left[L_{\ell, \tau}^{\tau}\right]_{(y, z)}^{\prime}(y, z, u, v, p, q)=0$. Hence, in the classical formulation, $p_{\ell}$ and $q_{\ell}$ are to solve the following system of linear elliptic boundary value problems:

$$
p^{N+1}=0, \quad q^{N+1}=0,
$$

and for $i=N, N-1, \cdots, 1$,

$$
\left\{\begin{array}{cc}
-\frac{p^{i+1}-p^{i}}{\tau}-d_{1} \Delta p^{i}+\left(\frac{b z^{i-1}}{1+e y^{i-1}}+y^{i-1} \frac{a}{k}\right) p^{i}-\left(\frac{d z^{i}}{1+e y^{i}}-\frac{d e z^{i} y^{i}}{\left(1+e y^{i}\right)^{2}}\right) q^{i} & \\
=p^{i+1} a\left(1-\frac{y^{i+1}}{k}\right)+b p^{i+1} \frac{e y^{i+1} z^{i}}{\left(1+e y^{i}\right)^{2}}+\beta_{1 \ell} D^{2 \alpha}\left(y^{i}-y_{\ell, d}^{i}\right) & \text { in } \Omega \\
-\frac{q^{i+1}-q^{i}}{\tau}-d_{2} \Delta q^{i}+\left(c-d \frac{y^{i}}{1+e y^{i}}\right) q^{i}=-p^{i+1} \frac{b y^{i+1}}{1+e y^{i}}+\beta_{2 \ell} D^{2 \alpha}\left(z^{i}-z_{\ell, d}^{i}\right) & \text { in } \Omega \\
p^{i}=0, \quad q^{i}=0 & \text { on } \Gamma
\end{array}\right.
$$


note that the undefined values of $y^{N+1}$ and $z^{N+1}$ are, in fact, irrelevant because of (60).

Remark 4.6. We point out that the above system of equations is a time discretization of the adjoint system (17). Furthermore, if we know $\left\{y^{k}\right\}_{k=0}^{N},\left\{z^{k}\right\}_{k=0}^{N}$, at each step we have to solve two uncoupled elliptic problems.

By the standard adjoint-equation technique, we have the formula for the Gâteaux differential of the payoffs:

$$
\begin{aligned}
{\left[\Phi_{\ell, \tau}\right]^{\prime}(u, v) } & =\left[L_{\ell, \tau}\right]_{(u, v)}^{\prime}\left(y, z, u, v, p_{\ell}, q_{\ell}\right)=\left((2-\ell) u^{r-1}+p_{\ell}^{1}\right. \\
& \left.+\tau p_{\ell}^{1}\left(a\left(1-\frac{y^{1}}{k}\right)+\frac{b e y^{1} v}{(1+e u)^{2}}\right),(\ell-1) v^{r-1}+q_{\ell}^{1}-\tau p_{\ell}^{1} \frac{b y^{1}}{1+e u}\right)
\end{aligned}
$$

with $(y, z) \in H_{0}^{1}(\Omega)^{2 N}$ being the response on $(u, v)$ and $\left(p_{\ell}, q_{\ell}\right)$ solving the above system.

\section{Full DisCRETIZATION}

Still a spatial discretization of the recursive elliptic boundary-value problems (37) has to be done. For this, we use the finite-element method with piecewise affine base functions. We do not perform the (lengthy and technical) analysis of the corresponding fully discrete case, mentioning only that Proposition 4.5 can be extended for this case, too.

We have to solve the following system of linear algebraic equations:

$$
y^{0}=u, \quad z^{0}=v,
$$

and for $i=1,2, \cdots, N$,

$$
\left\{\begin{array}{l}
B \frac{y^{i}-y^{i-1}}{\tau}+d_{1} C y^{i}=a B y^{i-1}-\frac{a}{k} B_{n l}\left(y^{i-1}\right) y^{i}-b B_{n l}\left(\frac{z^{i-1}}{1+e y^{i-1}}\right) y^{i} \\
B \frac{z^{i}-z^{i-1}}{\tau}+d_{2} C z^{i}=-c B z^{i}+d B_{n l}\left(\frac{y^{i}}{1+e y^{i}}\right) z^{i}
\end{array}\right.
$$

where $y^{i} \in \mathbb{R}^{\mathrm{I} \times 1}, i=0,1, \cdots, N$ are the coordinates of the fully discrete solution in the nodal finite element basis ( $I$ denotes the dimension of such a basis), $B \in \mathbb{R}^{\mathrm{I} \times \mathrm{I}}$ is the mass matrix, $C \in \mathbb{R}^{\mathrm{I} \times \mathrm{I}}$ is the stiffness matrix and $B_{n l}(\cdot): \mathbb{R}^{\mathrm{I} \times 1} \rightarrow \mathbb{R}^{\mathrm{I} \times \mathrm{I}}$ is a function providing suitable symmetric matrices for the nonlinear terms.

We approximate then the Nash Equilibrium problem (36) by the following fully discretized problem (we consider here the case $\alpha=0$ ):

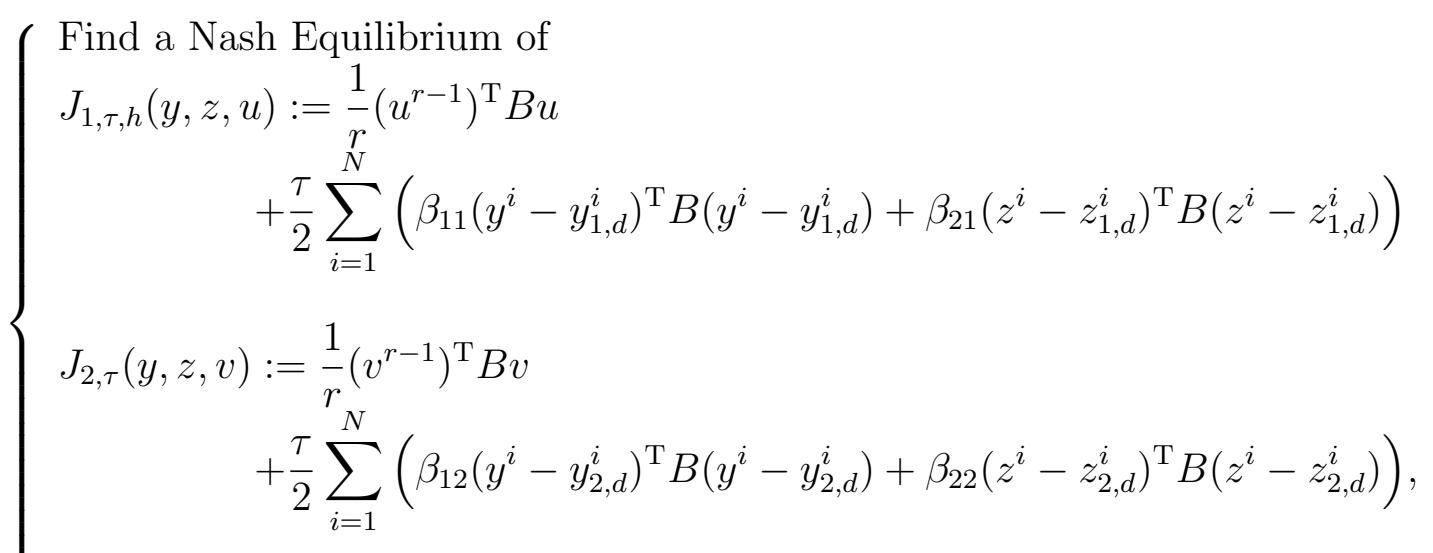

where $(u, v) \in \mathcal{U}_{h} \times \mathcal{V}_{h}$ and $y=\left\{y^{i}\right\}_{i=1}^{N}$ and $z=\left\{z^{i}\right\}_{i=1}^{N}$ solve (63)-(64), 
where $u^{r-1}$ denotes the vector in $\mathbb{R}^{\mathrm{I} \times 1}$ with all elements equal to the elements of vector $u$ to the power $r-1$ (and analogously for $v^{r-1}$ ).

For the fully discretized problem, the Lagrange function for the $\ell$-th player is the function

$$
L_{\ell, \tau, h}:\left(\mathbb{R}^{\mathrm{I} \times 1}\right)^{\mathrm{N}} \times\left(\mathbb{R}^{\mathrm{I} \times 1}\right)^{\mathrm{N}} \times \mathbb{R}^{\mathrm{I} \times 1} \times \mathbb{R}^{\mathrm{I} \times 1} \times\left(\mathbb{R}^{1 \times \mathrm{I}}\right)^{\mathrm{N}} \times\left(\mathbb{R}^{1 \times \mathrm{I}}\right)^{\mathrm{N}} \longrightarrow \mathbb{R}
$$

defined, for $y \in\left(\mathbb{R}^{\mathrm{I} \times 1}\right)^{\mathrm{N}}$ such that $y_{k}^{j} \geq 0, j=1, \cdots, N, k=1, \cdots, I$, by

$$
\begin{aligned}
L_{\ell, \tau, h}(y, z, u, v, p, q):=(2-\ell) \frac{1}{r}\left(u^{r-1}\right)^{\mathrm{T}} B u+(\ell-1) \frac{1}{r}\left(v^{r-1}\right)^{\mathrm{T}} B v \\
\quad+\frac{\tau}{2} \sum_{i=1}^{N}\left(\beta_{1 \ell}\left(y^{i}-y_{\ell, d}^{i}\right)^{\mathrm{T}} B\left(y^{i}-y_{\ell, d}^{i}\right)+\beta_{2 \ell}\left(z^{i}-z_{\ell, d}^{i}\right)^{\mathrm{T}} B\left(z^{i}-z_{\ell, d}^{i}\right)\right) \\
\quad-\tau\left(p^{1} B \frac{y^{1}-u}{\tau}+d_{1} p^{1} C y^{1}-a p^{1} B u+\frac{a}{k} p^{1} B_{n l}(u) y^{1}+b p^{1} B_{n l}\left(\frac{v}{1+e u}\right) y^{1}\right) \\
\quad-\tau \sum_{i=2}^{N}\left(p^{i} B \frac{y^{i}-y^{i-1}}{\tau}+d_{1} p^{i} C y^{i}-a p^{i} B y^{i-1} p^{i} B_{n l}\left(y^{i-1}\right) y^{i}+b p^{i} B_{n l}\left(\frac{z^{i-1}}{1+e y^{i-1}}\right) y^{i}\right) \\
\quad-\tau\left(q^{1} B \frac{z^{1}-v}{\tau}+d_{2} q^{1} C z^{1}+c q^{1} B z^{1}-d q^{1} B_{n l}\left(\frac{y^{1}}{1+e y^{1}}\right) z^{1}\right) \\
\quad-\tau \sum_{i=2}^{N}\left(q^{i} B \frac{z^{i}-z^{i-1}}{\tau}+d_{2} q^{i} C z^{i}+c q^{i} B z^{i}-d q^{i} B_{n l}\left(\frac{y^{i}}{1+e y^{i}}\right) z^{i}\right) .
\end{aligned}
$$

The adjoint system for the $\ell$-th player is $\left[L_{\ell, \tau}\right]_{(y, z)}^{\prime}(y, z, u, v, p, q)=0$. Let us denote $p=p_{l}$ and $q=q_{l}$ to the solution of $\left[L_{\ell, \tau, h}^{\tau}\right]_{(y, z)}^{\prime}(y, z, u, v, p, q)=0$. Hence, in the classical formulation, $p_{\ell}$ and $q_{\ell}$ are to solve the following system of linear algebraic equations:

$$
p^{N+1}=0, \quad q^{N+1}=0,
$$

and for $i=N, N-1, \cdots, 1$,

$$
\left\{\begin{array}{r}
-\frac{p^{i+1}-p^{i}}{\tau} B+d_{1} p^{i} C+\frac{a}{k} p^{i} B_{n l}\left(y^{i-1}\right)+b p^{i} B_{n l}\left(\frac{z^{i-1}}{1+e y^{i-1}}\right) \\
-d \nabla_{y^{i}}\left(q^{i} B_{n l}\left(\frac{y^{i}}{1+e y^{i}}\right) z^{i}\right)=a p^{i+1} B-\frac{a}{k} \nabla_{y^{i}}\left(p^{i+1} B_{n l}\left(y^{i}\right) y^{i+1}\right) \\
-b \nabla_{y^{i}}\left(p^{i+1} B_{n l}\left(\frac{z^{i}}{1+e y^{i}}\right) y^{i+1}\right)+\beta_{1 \ell}\left(y^{i}-y_{\ell, d}^{i}\right)^{\mathrm{T}} B \\
-\frac{q^{i+1}-q^{i}}{\tau} B+d_{2} q^{i} C+c q^{i} B-d q^{i} B_{n l}\left(\frac{y^{i}}{1+e y^{i}}\right) \\
=-b \nabla_{z^{i}}\left(p^{i+1} B_{n l}\left(\frac{z^{i}}{1+e y^{i}}\right) y^{i+1}\right)+\beta_{2 \ell}\left(z^{i}-z_{\ell, d}^{i}\right)^{\mathrm{T}} B
\end{array}\right.
$$

note that the undefined values of $y^{N+1}$ and $z^{N+1}$ are, in fact, irrelevant because of (66).

Remark 5.1. We point out that the above system of equations is a space discretization of the adjoint time discretized system $60-61$ and therefore, a full discretization of the 
adjoint system (17). Furthermore, if we know $\left\{y^{k}\right\}_{k=0}^{N},\left\{z^{k}\right\}_{k=0}^{N}$, at each step we have to solve two uncoupled algebraic problems.

By the standard adjoint-equation technique, we have the formula for the differential of the payoffs:

$$
\begin{gathered}
\left((2-\ell)\left(u^{r-1}\right)^{\mathrm{T}} B+p_{\ell}^{1} B+a \tau p_{\ell}^{1} B-\frac{a}{k} \tau \nabla_{u}\left(p_{\ell}^{1} B_{n l}(u) y^{1}\right)-b \tau \nabla_{u}\left(p_{\ell}^{1} B_{n l}\left(\frac{v}{1+e u}\right) y^{1}\right)\right. \\
\left.(\ell-1)\left(v^{r-1}\right)^{\mathrm{T}} B+q_{\ell}^{1} B-b \tau \nabla_{v}\left(p_{\ell}^{1} B_{n l}\left(\frac{v}{1+e u}\right) y^{1}\right)\right)
\end{gathered}
$$

with $(y, z) \in\left(\mathbb{R}^{\mathrm{I} \times 1}\right)^{N} \times\left(\mathbb{R}^{\mathrm{I} \times 1}\right)^{N}$ being the response on $(u, v)$ in question and $\left(p_{\ell}, q_{\ell}\right)$ solves the above system.

\section{ILLUSTRATIVE NUMERICAL EXAMPLES}

6.1. A controlled system and a game. For computational simulations, we consider the one-dimensional test problem given by $\Omega=(0,1), a=1, b=0.5, c=3, d=.5, e=0.05$, $k=20, d_{1}=0.1$, and $d_{2}=0.2$. With these data, the associated Lotka-Volterra type ODE system (i.e., in the absence of diffusion and boundary conditions) has a coexistence equilibrium given by $\left(y_{\mathrm{s}}, z_{\mathrm{s}}\right) \approx(8.56,1.6379)$ (see Figure 1$)$.

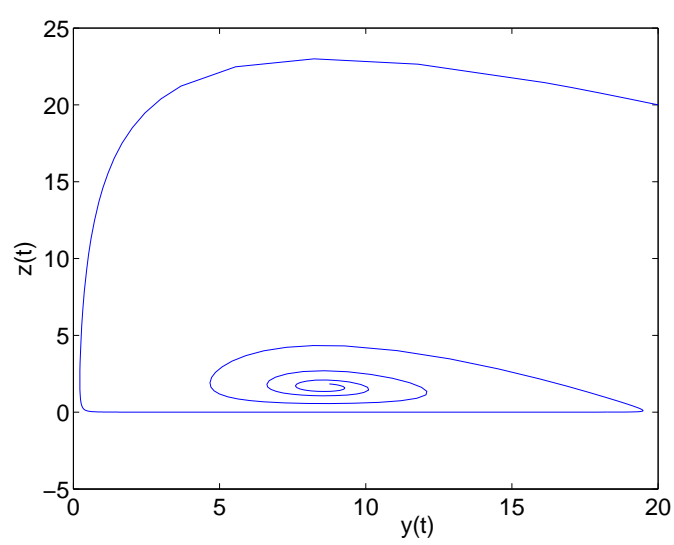

Figure 1. Phase diagram of the associated ODE system, for $t \in[0,30]$.

The data that we have taken for the functionals involved in the Nash equilibrium problem are: $T=30, r=2, y_{1, \mathrm{~d}}=8.56, z_{1, \mathrm{~d}}=0, y_{2, \mathrm{~d}}=0, z_{2, \mathrm{~d}}=1.6379, \beta_{11}=1$, $\beta_{22}=100, \beta_{21}=\beta_{12}=0, \alpha=0$ and

$$
u_{\max }(x)=\left\{\begin{array}{ll}
20 & \text { if } 0.2<x<0.4, \\
0 & \text { elsewhere }
\end{array} \quad \text { and } \quad v_{\max }(x)= \begin{cases}20 & \text { if } 0.6<x<0.8 \\
0 & \text { elsewhere }\end{cases}\right.
$$

Therefore the players 1 and 2 want the response to be close to $y_{\mathrm{s}}$ and $z_{\mathrm{s}}$, respectively, taking also into account the cost of the implementation of their controls (in this case the cost of the preys is much more expensive than that of the predators). 
Verification of the assumptions of Proposition 3.6 is however very difficult, and we do not know whether the above data satisfy them, and henceforth we will proceed rather "blindly".

6.2. Nash equilibrium search algorithm. The existence of a constructive method to solve the corresponding finite-dimensional game remains, to the best of our knowledge, an open problem for general cases. In [21] a converging conjugate-gradient type algorithm has been used for linear partial differential equations. For nonlinear systems with suitable convex properties of the functionals involved (as those showed above) an iterative procedure has been developed in [12], but it requires the minimization of a non-convex function over compact domains, which is not well-suited for an implementation on the computer.

Anyhow, we propose the following conceptual algorithm to compute an approximate solution of the Nash equilibrium problem:

Step 1. $\left(u_{0}, v_{0}\right)$ is given in $\mathcal{U}_{h} \times \mathcal{V}_{h}, k:=0$.

Step 2. Find $u_{k+1} \in \operatorname{Argmin}_{\mathcal{U}_{h}} \Phi_{1, \tau}\left(\cdot, v_{k}\right)$.

Step 3. Find $v_{k+1} \in \operatorname{Argmin}_{\mathcal{V}_{h}} \Phi_{2, \tau}\left(u_{k+1}, \cdot\right)$.

Step 4. If

$$
\frac{\left\|u_{k+1}-u_{k}\right\|_{L^{r}}}{\max \left\{\left\|u_{k+1}\right\|_{L^{r}},\left\|u_{k}\right\|_{L^{r}}, 1\right\}} \leq \text { factr } * \text { eps }
$$

and

$$
\frac{\left\|v_{k+1}-v_{k}\right\|_{L^{r}}}{\max \left\{\left\|v_{k+1}\right\|_{L^{r}},\left\|v_{k}\right\|_{\left.L^{r}, 1\right\}}\right.} \leq \text { factr } * \text { eps }
$$

then stop else do $k:=k+1$ and go to Step 2 .

After the stop, $\left(u_{k+1}, v_{k+1}\right)$ is to be considered as an approximate Nash equilibrium. In Step 4, eps and factr denote the machine precision of the computer and a control factor for the stopping criteria, respectively. The value of eps can be taken directly from the computer (typically eps $\approx 10^{-16}$ ). Typical values for factr are: $10^{12}$ for low accuracy, $10^{7}$ for moderate accuracy and 10 for extremely high accuracy. In all our numerical tests we have used a personal computer with eps $\approx 2.220410^{-16}$ and a control factor factr $=10^{7}$.

We point out that $u_{0}$ is seemingly unimportant but it is implicitly to be used as a starting iteration for the iterative procedure solving the minimization problem in Step 2 for $k=0$. Moreover, we point out that there is no proof of stopping of the above iterative procedure for a finite $k$ (we can even show a counterexample) even if some Nash equilibrium does exist, but it follows a natural way which real systems are tempted for reaching an equilibrium (although it does not always lead to it). That is why we expect our algorithm to be convergent at least in "naturally qualified" situations. This procedure has already been used (in a very similar form) in [22] and [20] for a pointwise control of the Burgers equation.

To compute numerically a Nash equilibrium we have used the time discretization scheme showed above, with $N=300$, we have fully discretized the problem by using a Finite Element Method, with linear finite elements of size $h=1 / 50$ and we have used the above proposed algorithm with factr $=10^{7}$. Of course, the minimization of $\Phi_{1, \tau}(\cdot, v)$ and of $\Phi_{2, \tau}(u, \cdot)$ can be solved only approximately because these functionals are not quadratic unless a trivial non-interesting case $a=b=d=0$. Here we use a subspace trust region method, which is based on the interior-reflective Newton method described in [3], [4]. 
This method requires only the values of $\Phi_{1, \tau}$ and $\Phi_{2, \tau}$ and their first derivatives $\frac{\partial}{\partial u} \Phi_{1, \tau}$ and $\frac{\partial}{\partial v} \Phi_{2, \tau}$ given by the full discretization of (62).

6.3. Numerical test. For the numerical test we have used $N=300$ and $I=49$.

If we start with $u_{0} \equiv 8.56$ on $(0.2,0.4)$ and $v_{0} \equiv 1.6379$ on $(0.6,0.8)$, Figures $2-3$ show the solutions $(y, z)$ for the corresponding initial conditions $(u, v):=\left(u_{0}, v_{0}\right)$, still without minimizing any functional. They show the typical oscillatory solutions of the Lotka-Volterra type systems, around the coexistence equilibrium.

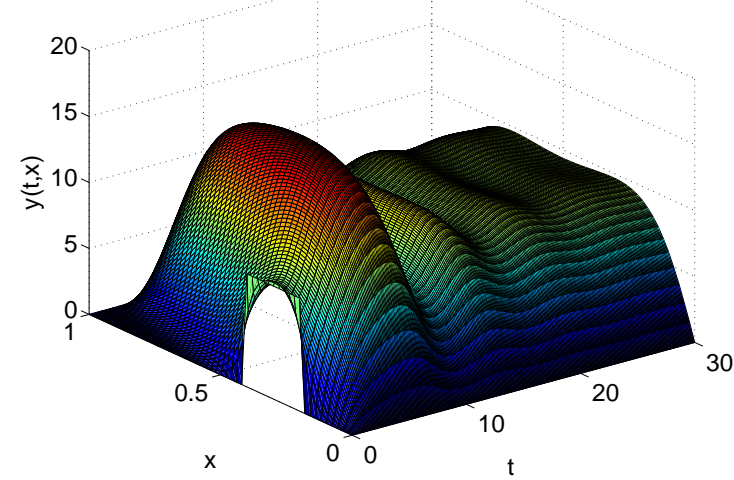

FiguRE 2. Graphic of $y(t, x)$ when $u=u_{0} \equiv 8.56$ on $(0.2,0.4)$ and $v=v_{0} \equiv 1.6379$ on $(0.6,0.8)$.

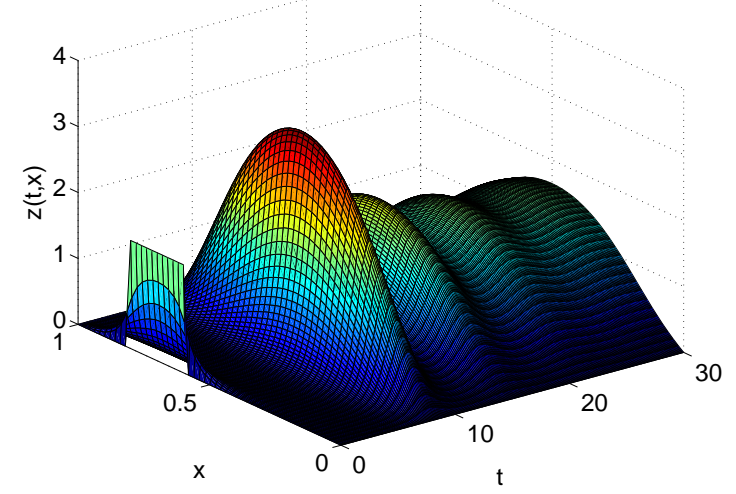

FiguRE 3. Graphic of $z(t, x)$ when $u \equiv 8.56$ on $(0.2,0.4)$ and $v \equiv 1.6379$ on $(0.6,0.8)$.

Figures $4-5$ show the solutions $(y, z)$ for the initial conditions $(u, v):=\left(u_{6}, v_{6}\right)$ corresponding to the computed Nash equilibrium.

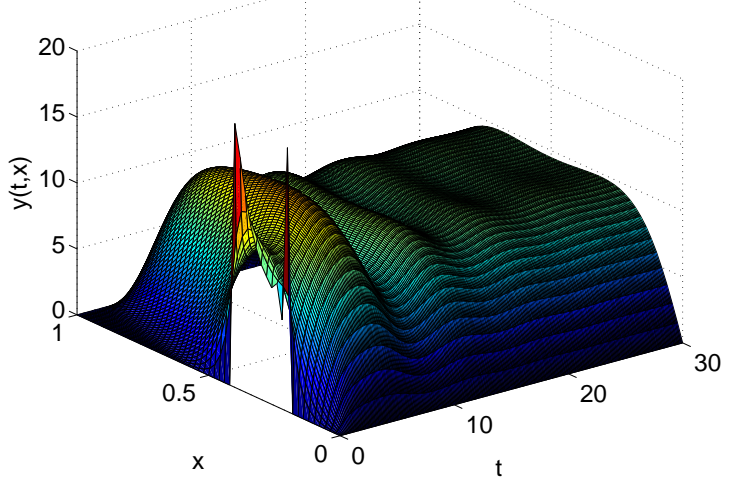

FiguRE 4. Graphic of $y(t, x)$ when $(u, v)=\left(u_{6}, v_{6}\right)$ are the computed Nash equilibrium on $(0.2,0.4)$.

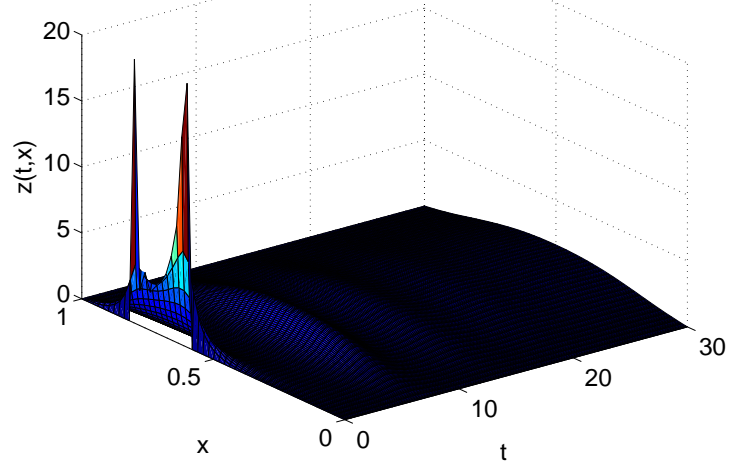

FiguRE 5. Graphic of $z(t, x)$ when $(u, v)=\left(u_{6}, v_{6}\right)$ are the computed Nash equilibrium on $(0.6,0.8)$.

Figures 6-7 show the graphs of $\left\|y(t)-y_{1, \mathrm{~d}}\right\|_{2}$ and $\left\|z(t)-z_{2, \mathrm{~d}}\right\|_{2}$, respectively, where $(y, z)$ are the solution corresponding to the initial conditions $\left(u_{0}, v_{0}\right)$ (the full line: - ) and to $\left(u_{6}, v_{6}\right)$ (the dashed line: - - -).

Table 1 shows the value of the payoff functionals at each iteration. 


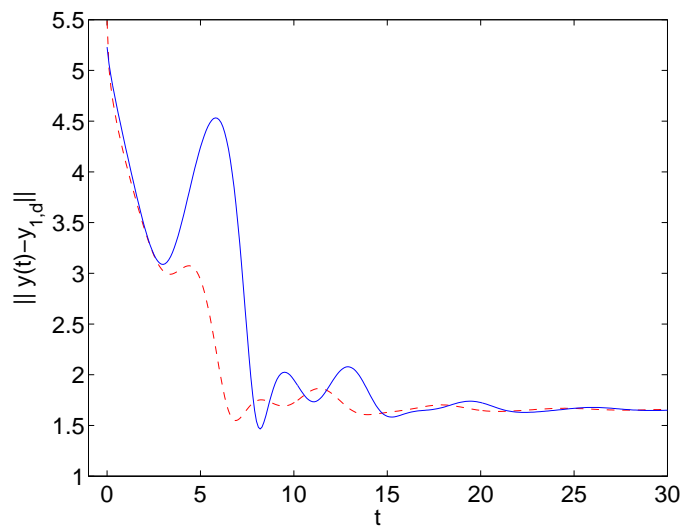

FiguRE 6. Graphic of $\left\|y(t)-y_{1, \mathrm{~d}}\right\|_{2}$ for $\left(u_{0}, v_{0}\right)(-)$ and $\left(u_{6}, v_{6}\right)(--)$.

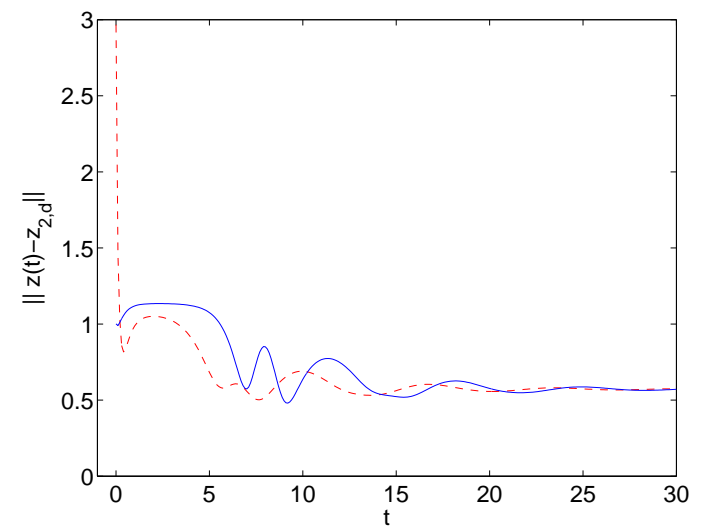

FIGURE 7. Graphic of $\left\|z(t)-z_{2, \mathrm{~d}}\right\|_{2}$ for $\left(u_{0}, v_{0}\right)(-)$ and $\left(u_{6}, v_{6}\right)(---)$.

\begin{tabular}{|l|l|l|}
\hline & $\Phi_{1}$ & $\Phi_{2}$ \\
\hline \hline $\mathrm{k}=0$ & 187.6703 & $1.6118 \mathrm{e}+3$ \\
\hline $\mathrm{k}=1$ & 176.3443 & $1.3203 \mathrm{e}+3$ \\
\hline $\mathrm{k}=2$ & 152.6978 & $1.3223 \mathrm{e}+3$ \\
\hline $\mathrm{k}=3$ & 152.6806 & $1.3224 \mathrm{e}+3$ \\
\hline $\mathrm{k}=4$ & 152.6799 & $1.3224 \mathrm{e}+3$ \\
\hline $\mathrm{k}=5$ & 152.6799 & $1.3224 \mathrm{e}+3$ \\
\hline $\mathrm{k}=6$ & 152.6799 & $1.3224 \mathrm{e}+3$ \\
\hline
\end{tabular}

TABLE 1. Value of the payoff functionals at each iteration.

Acknowledgment: A.M. Ramos was supported by the MCyT of Spain under a "Ramón y Cajal" research contract, and by the 'Plan Nacional de I+D+I (2000-2003)' of the MCyT (Spain), through the AGL2003-06862-C02-02 project. T. Roubíček was partly supported by the grants 201/03/0934 (GA ČR) and MSM 0021620839 (MŠMT ČR).

\section{REFERENCES}

[1] Arndt M, Griebel M, Roubíček T (2003) Modelling and numerical simulation of martensitic transformation in shape memory alloys. Continuum Mech Thermodyn 15: 463-485.

[2] Britton NF (1986) Reaction-diffusion equations and their applications to biology. Acad. Press, London.

[3] Coleman TF, Li Y (1994) On the Convergence of Reflective Newton Methods for Large-Scale Nonlinear Minimization Subject to Bounds. Mathematical Programming 67/2: 189-224.

[4] Coleman TF, Li Y (1996) An Interior, Trust Region Approach for Nonlinear Minimization Subject to Bounds. SIAM Journal on Optimization 6: 418-445.

[5] Díaz JI, Henry J, Ramos AM (1998) On the approximate controllability of some semilinear parabolic boundary value problems. Appl Math Opt 37/1: 71-97.

[6] Díaz JI, Ramos AM (1997) Some Results About the Approximate Controllability Property for Quasilinear Diffusion Equations. C R Acad Sci I-Math, t. 324, Série I, 1243-1248.

[7] Díaz JI, Ramos AM (2004) Numerical Experiments Regarding the Distributed Control of Semilinear Parabolic Problems. Computers \& Mathematics with Applications 48: 1575-1586.

[8] Freedman HI (1980) Deterministic Mathematical Models in Population Ecology. Dekker, New York. 
[9] Gabasov R, Kirillova F (1976) The Qualitative Theory of Optimal Processes. Marcel Dekker, New York (Russian orig.: Nauka, Moscow, 1971).

[10] Glowinski R, Ramos AM (2002) A Numerical Approach to the Neumann Control of the CahnHilliard Equation. In Computational Methods for Control and Applications. Eds.: R. Glowinski, H. Kawarada and J. Periaux, Gakuto International Series: Mathematical Sciences and Applications, Vol. 16, Gakkotosho Co., Tokyo, pp. 111-155.

[11] Huang J, Lu G, Ruan S (2003) Existence of traveling wave solutions in a diffusive predator-prey model. J Math Biology 46: 132-152.

[12] Iusem A, Sosa W (2003) Iterative Algorithms for Equilibrium Problems. Optimization 52: 301-316.

[13] Leung A (1982) A semilinear reaction-diffusion predator-prey system with nonlinear coulpled boundary conditions. Indiana Univ Math J 31: 223-241.

[14] Lotka A (1920) Undamped oscillations derived from the law of mass action. J Am Chem Soc 42: $1595-1599$

[15] May R (1974) Stablity and Complexity in Model Ecosystems. Princeton Univ. Press, Princetown.

[16] Murray JD (1989) Mathemacal Biology. Springer, Berlin.

[17] Nash J (1951) Non-cooperative games. Annals of Math 54: 286-295.

[18] Nikaidô H, Isoda K (1955) Note on non-cooperative equilibria. Pacific J Math 5: 807-815.

[19] Pao VC (1992) Nonlinear Parabolic and Elliptic Equations. Plenum Press, New York, 1992.

[20] Ramos AM (2003) Numerical Methods for Nash Equilibria in Multiobjective Control of Partial Differential Equations. In Analysis and Optimization of Differential Systems. Eds.: V. Barbu, I. Lasiecka, D. Tiba and C. Varsan. Kluwer, Boston, pp. 333-344.

[21] Ramos AM, Glowinski R, Periaux J (2002) Nash Equilibria for the Multi-Objective Control of Linear Partial Differential Equations. J Optimiz Theory App 112/3: 457-498.

[22] Ramos AM, Glowinski R, Periaux J (2002) Pointwise Control of the Burgers Equation and related Nash Equilibrium Problems: A Computational Approach. J Optimiz Theory App 112/3: 499-516.

[23] Roubíček T (1997) Relaxation in Optimization Theory and Variational Calculus. W. de Gruyter, Berlin.

[24] Roubíček T (1999) On noncooperative nonlinear differential games. Kybernetika 35: 487-498.

[25] Roubíček T (1999) Noncooperative games with elliptic systems. In Proc. IFIP WG 7.2 Conf. Optimal Control of P.D.E. (Eds. K.-H.Hoffmann, G.Leugering, F.Tröltzsch), ISNM 133, Birkhäuser, Basel, pp. $245-255$.

[26] Roubíček T (2003) Optimization of steady-state flow of incompressible fluids. In Analysis and Optimization of Differential Systems. Eds.: V. Barbu, I. Lasiecka, D. Tiba and C. Varsan. Kluwer, Boston, pp. 357-368.

[27] Roubíček T (to appear in 2005) Nonlinear Partial Differential Equations and Applications. Birkhäuser, Basel.

[28] Volterra V (1926) Variazionie fluttuaziooni del numero d'individui in specie animali conviventi. Mem Acad Lincei 2: 31-113.

[29] Williams S, Chou PL (1978) Nonlinear raection-diffusion models for interacting populations. J Math Anal Appl 62: 157-169. 